\title{
SURFACE WAVES IN MULTILAYERED ELASTIC MEDIA I. RAYLEIGH AND LOVE WAVES FROM BURIED SOURCES IN A MULTILAYERED ELASTIC HALF-SPACE
}

\author{
By David G. Harkrider
}

\begin{abstract}
A matrix formulation is used to derive integral expressions for the time transformed displacement fields produced by simple sources at any depth in a multilayered elastic isotropic solid half-space. The integrals are evaluated for their residue contribution to obtain surface wave displacements in the frequency domain. The solutions are then generalized to include the effect of a surface liquid layer. The theory includes the effect of layering and source depth for the following: (1) Rayleigh waves from an explosive source, (2) Rayleigh waves from a vertical point force, (3) Rayleigh and Love waves from a vertical strike slip fault model. The latter source also includes the effect of fault dimensions and rupture velocity. From these results we are able to show certain reciprocity relations for surface waves which had been previously proved for the total displacement field. The theory presented here lays the ground work for later papers in which theoretical seismograms are compared with observations in both the time and frequency domain.
\end{abstract}

\section{INTRODUCTION}

Several years ago Dorman, Ewing, and Oliver (1960) successfully utilized the Thomson-Haskell matrix formulation (Haskell, 1953) in the calculation of surface wave dispersion on multilayered elastic media using a high speed computer. Since that time surface wave dispersion has been used extensively in the interpretation of the earth's structure. Calculation of dispersion had previously been limited to simple earth models consisting of at most three layers.

The success of surface wave dispersion in yielding additional knowledge on the earth's upper mantle structure and on the earthquake source mechanism has given hope to seismologists that the amplitude spectra of surface waves may provide further information concerning the mechanism of seismic sources. This is especially true for source depth which has no influence on dispersion. In order to determine the effect of source location on surface wave amplitudes it is necessary to include a source at depth in the multilayered formulation. Also without a specific source one is unable to determine the relative excitation between modes as a function of frequency.

There are two methods of attacking the source problem for an $n$-layered medium. The classical technique uses the determinants that result from Cramer's rule for solving a set of inhomogeneous linear equations. One expresses the source as an integration of homogeneous solutions to which have been added homogeneous layer solutions with arbitrary coefficients so as to be able to satisfy the boundary conditions at each interface. In this way one arrives at a formal integral solution with an integrand given in terms of the ratios of two determinants of order $(4 n-2)$ for Rayleigh waves and $(2 n-2)$ for Love waves.

This method, although easy to formulate, is extremely cumbersome if not dangerous to evaluate numerically on a computer. The danger involved results from the fact that determinants in general are not slowly varying functions of their elements. 
The determinant solution was obtained by Jardetzky (1953) and Kellis-Borok (1953). Besides the numerical difficulties inherent in solving large order determinants there is the practical difficulty of reordering or simplifying the determinants into a form which provides insight into the individual effects of receiver depth, source depth, and layering on the spectral amplitude.

The second method is to use a matrix formulation. Previously this has been done in two ways. Using the Thomson-Haskell matrices to obtain the reflection and transmission coefficients for plane waves in multilayered media (Thomson, 1950), and an integral representation of a point source in terms of plane waves, Gilbert (1956) obtained a formal integral solution for the compressional point source, but made no effort to evaluate the integral for the surface wave contribution.

Gilbert and MacDonald (1961) applied the Thomson-Haskell matrix method to a layered sphere using the solutions of the equations of motion for an elastic shell. They obtained the solution to the source-at-depth problem for the sphere by operating on the source vector equation with a matrix product of the shell matrices. The source vector equation was obtained by evaluating the source at positions infinitesimally above and below the source depth.

This paper derives in detail an integral solution for the time transformed displacements for certain elementary sources at depth in a multilayered isotropic halfspace. The integrands are expressed in terms of elements from the matrix product of the Thomson-Haskell layer matrices in the layered array. These integrands are obtained by a technique similar to that used by Gilbert and MacDonald, namely, by a matrix operation on a general source vector equation. The elements of the vector equation depend on the integrand of the particular type of source under investigation. The transformed sources considered are as follows: (1) An explosive or spherical pressure source, (2) A horizontal and vertical point force, (3) A model of vertical strike slip fault sources formed by integration of a time lagged horizontal singlet or doublet point force over the fault surface.

The vertical and horizontal point forces are not as restrictive as one might surmise. Their generality was shown by Kellis-Borok (1953) who pointed out that the field due to a point force, $F$, of arbitrary direction in a multilayered media can be obtained by the superposition of the fields due to a vertical point force, $F \sin \Delta$, and a horizontal point force, $F \cos \Delta$, where $\Delta$ is the vertical angle between $F$ and the horizontal. Furthermore, displacement fields for multipole sources can be determined by spatial differentiation.

From the residue contribution of the integral solutions, we obtain the Rayleigh and Love wave displacements for various source types. If we had stopped here this problem would have been merely an extension of the matrix technique of Gilbert and MacDonald to Rayleigh and Love waves on a multilayered media for different types of sources. Extensive programming and numerical analysis would have been needed to compute amplitude spectra or theoretical seismograms. However by obtaining a simple form of the inverse of the product matrix in terms of the elements of the product matrix itself and simplifying the residue numerator we are able to separate the solution into factors representing source depth, receiver depth, layering, and path of propagation. The necessary simplification of the numerator is accomplished by using relations determined by setting the integrand denominator 
to zero. The factors representing source and receiver depth are shown to be simple functions of quantities calculated in the plane wave problem. Using the numerical techniques to be described in Part II the excitation function for the layered medium can be calculated analytically by simple modifications of computer programs which are currently used in seismology to calculate dispersion for Rayleigh and Love surface waves.

\section{The Equations of Motion and the Matrix Relations for layers Not Contalning a Source}

First we consider a semi-infinite elastic medium made up of $n$ parallel, solid, homogeneous, isotropic layers (figure 1). We number the array such that the layer at the free surface is layer 1 and the half-space is layer $n$. Placing the origin of a cylindrical coordinate system $(r, \theta, z)$ at the free surface, the layer interfaces are defined by $z$ constant and any given layer $m$ is bounded by $z_{m-1}$ and $z_{m}$ with $z_{m}>$ $z_{m-1}$.

The Fourier time transformed vector equation of motion for an isotropic elastic solid $m$ is

$$
\left(\lambda_{m}+2 \mu_{m}\right) \operatorname{grad} \operatorname{div} \hat{S}_{m}-\mu_{m} \operatorname{curl}\left(\operatorname{curl} \hat{S}_{m}\right)=-\omega^{2} \rho_{m} \hat{S}_{m}
$$

where the following notation has been used:

$$
\begin{aligned}
& \hat{S}_{m}=\left(\bar{q}_{m}, \bar{v}_{m}, \bar{w}_{m}\right) ; \text { the displacement vector with }(r, \theta, z) \text { components } \\
& \omega ; \text { angular frequency } \\
& \rho_{m} ; \text { density } \\
& \lambda_{m}, \mu_{m} ; \text { Lame's constants } \\
& \alpha_{m}=\left(\frac{\lambda_{m}+2 \mu_{m}}{\rho_{m}}\right)^{1 / 2} ; \text { compressional wave velocity } \\
& \beta_{m}=\left(\frac{\mu_{m}}{\rho_{m}}\right)^{1 / 2} ; \text { shear wave velocity } \\
& k_{\alpha_{m}}=\frac{\omega}{\alpha_{m}} ; \text { compressional wave number } \\
& k_{\beta_{m}}=\frac{\omega}{\beta_{m}} ; \text { shear wave number }
\end{aligned}
$$

Defining the potentials $\bar{\varphi}, \bar{\psi}$, and $\bar{\chi}$ implicitly as 


$$
\begin{aligned}
& \bar{q}_{m}(r, \theta, z)=\frac{\partial \bar{\varphi}_{m}}{\partial r}+\frac{\partial^{2} \bar{\psi}_{m}}{\partial r \partial z}+\frac{1}{r} \frac{\partial \bar{\chi}_{m}}{\partial \theta} \\
& \bar{v}_{m}(r, \theta, z)=\frac{1}{r} \frac{\partial \bar{\varphi}_{m}}{\partial \theta}+\frac{1}{r} \frac{\partial^{2} \bar{\psi}_{m}}{\partial z \partial \theta}-\frac{\partial \bar{\chi}_{m}}{\partial r} \\
& \bar{w}_{m}(r, \theta, z)=\frac{\partial \bar{\varphi}_{m}}{\partial z}-\frac{1}{r} \frac{\partial}{\partial r}\left(r \frac{\partial \bar{\psi}_{m}}{\partial r}\right)+\frac{1}{r^{2}} \frac{\partial^{2} \bar{\psi}_{m}}{\partial \theta^{2}}
\end{aligned}
$$

which represent the transformed radial, azimuthal and vertical displacements

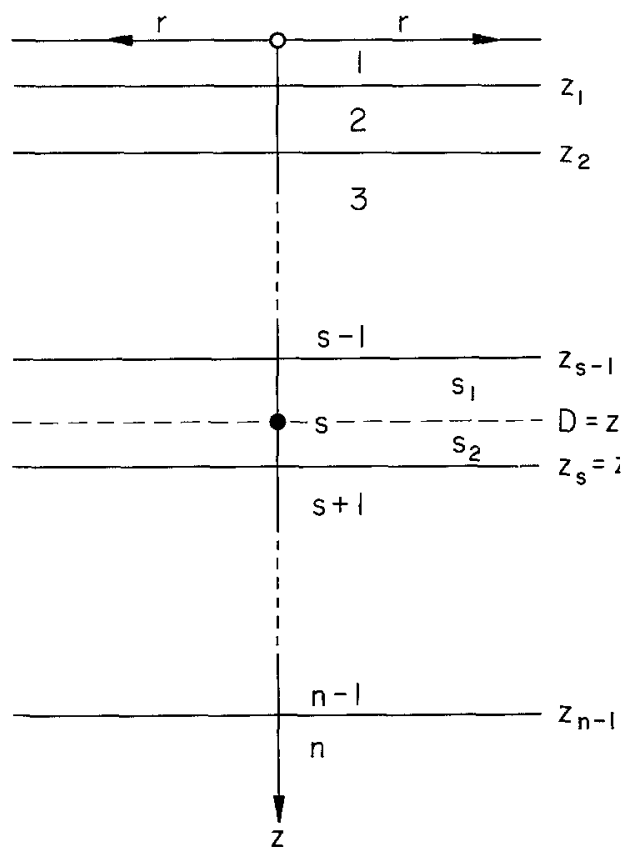

FIG. 1. Direction of axes, numbering of layers, and the depth of interfaces and source.

respectively, and substituting into equation (1) we see that

$$
\operatorname{div} \hat{S}_{m}=\nabla^{2} \bar{\varphi}_{m}
$$

and that equation (1) is satisfied if $\bar{\varphi}_{m}, \bar{\psi}_{m}$ and $\bar{\chi}_{m}$ are the solutions of

$$
\begin{aligned}
\nabla^{2} \bar{\varphi}_{m} & \equiv \frac{1}{r} \frac{\partial}{\partial r}\left(r \frac{\partial \bar{\varphi}_{m}}{\partial r}\right)+\frac{1}{r^{2}} \frac{\partial^{2} \bar{\varphi}_{m}}{\partial \theta^{2}}+\frac{\partial^{2} \bar{\varphi}_{m}}{\partial z^{2}}=-k_{\alpha_{m}}^{2} \bar{\varphi}_{m} \\
\nabla^{2} \bar{\psi}_{m} & =-k_{\beta_{m}}^{2} \bar{\psi}_{m} \\
\nabla^{2} \bar{\chi}_{m} & =-k_{\beta_{m}}^{2} \bar{\chi}_{m}
\end{aligned}
$$

Using (3) with (2) and the definitions of stress in terms of displacement, we obtain 
the normal, azimuthal tangential, and the radial tangential stress to the $z$ plane or $\bar{P}_{z z}, \bar{P}_{\theta_{z}}$, and $\bar{P}_{r z}$ respectively

$$
\begin{aligned}
& \bar{q}_{m}(r, \theta, z)=\frac{\partial \bar{\varphi}_{m}}{\partial r}+\frac{\partial^{2} \bar{\psi}_{m}}{\partial r \partial z}+\frac{1}{r} \frac{\partial \bar{\chi}_{m}}{\partial \theta} \\
& \bar{v}_{m}(r, \theta, z)=\frac{1}{r} \frac{\partial \bar{\varphi}_{m}}{\partial \theta}+\frac{1}{r} \frac{\partial^{2} \bar{\psi}_{m}}{\partial z \partial \theta}-\frac{\partial \bar{\chi}_{m}}{\partial r} \\
& \bar{w}_{m}(r, \theta, z)=\frac{\partial \bar{\varphi}_{m}}{\partial z}+\frac{\partial^{2} \bar{\psi}_{m}}{\partial z^{2}}+k_{\beta_{m}}^{2} \bar{\psi}_{m} \\
& \bar{P}_{z z_{m}}(r, \theta, z)=2 \mu_{m} \frac{\partial \bar{w}_{m}}{\partial z}+\lambda_{m} \operatorname{div} \hat{S}_{m} \\
& =2 \mu_{m}\left[\frac{\partial^{2} \bar{\varphi}_{m}}{\partial z^{2}}+\frac{\partial^{3} \bar{\psi}_{m}}{\partial z^{3}}+k_{\beta_{m}}^{2} \frac{\partial \bar{\psi}_{m}}{\partial z}-\lambda_{m} k_{\alpha_{m}}^{2} \bar{\varphi}_{m}\right] \\
& \bar{P}_{\theta z_{m}}(r, \theta, z)=\mu_{m}\left(\frac{1}{r} \frac{\partial \bar{w}_{m}}{\partial \theta}+\frac{\partial \bar{v}_{m}}{\partial z}\right) \\
& =\mu_{m}\left[\frac{2}{r^{r}} \frac{\partial^{2} \bar{\varphi}_{m}}{\partial z \partial \theta}+\frac{2}{r} \frac{\partial^{3} \bar{\psi}_{m}}{\partial z^{2} \partial \theta}+\frac{k_{\beta_{m}}^{2}}{r} \frac{\partial \bar{\psi}_{m}}{\partial \theta}-\frac{\partial^{2} \bar{\chi}_{m}}{\partial r \partial \theta}\right] \\
& \bar{P}_{r z_{m}}(r, \theta, z)=\mu_{m}\left(\frac{\partial \bar{w}_{m}}{\partial r}+\frac{\partial \bar{q}_{m}}{\partial z}\right) \\
& =\mu_{m}\left[2 \frac{\partial^{2} \bar{\varphi}_{m}}{\partial z \partial r}+2 \frac{\partial^{3} \bar{\psi}_{m}}{\partial z^{2} \partial r}+k_{\beta_{m}}^{2} \frac{\partial \bar{\psi}_{m}}{\partial r}+\frac{1}{\gamma} \frac{\partial^{2} \bar{\chi}_{m}}{\partial z \partial \theta}\right]
\end{aligned}
$$

In passing, we note that if $\bar{\chi}_{m}$ is independent of $r$ and if $\bar{\varphi}_{m}, \bar{\psi}_{m}$ and $\bar{\chi}_{m}$ are independent of $\theta$, equations (4) reduce to the usual potential expressions for cylindrical displacements involving azimuthal symmetry (Ewing, Jardetzky, and Press, 1957).

We now define

$$
\begin{array}{rlrl}
\bar{q}_{m}(r, \theta, z) & =\int_{0}^{\infty} q_{m}(r, \theta, z ; k) d k, & & \bar{P}_{z z_{m}}(r, \theta, z)=\int_{0}^{\infty} P_{z z_{m}}(r, \theta, z ; k) d k, \\
\bar{v}_{m}(r, \theta, z)=\int_{0}^{\infty} v_{m}(r, \theta, z ; k) d k, & \bar{P}_{\theta z_{m}}(r, \theta, z)=\int_{0}^{\infty} P_{\theta z_{m}}(r, \theta, z ; k) d k, \\
\bar{w}_{m}(r, \theta, z)=\int_{0}^{\infty} w_{m}(r, \theta, z ; k) d k, & \bar{P}_{r z_{m}}(r, \theta, z)=\int_{0}^{\infty} P_{r z_{m}}(r, \theta, z ; k) d k, \\
\bar{\varphi}_{m}(r, \theta, z)=\int_{0}^{\infty} \varphi_{m}(r, \theta, z ; k) d k, & \bar{\psi}_{m}(r, \theta, z)=\int_{0}^{\infty} \psi_{m}(r, \theta, z ; k) d k,
\end{array}
$$


and

$$
\bar{\chi}_{m}(r, \theta, z)=\int_{0}^{\infty} \chi_{m}(r, \theta, z ; k) d k .
$$

Assuming the following separable radial and azimuthal dependence for the potential integrands

$$
\begin{aligned}
& \varphi_{m}(r, \theta, z ; k)=\varphi_{m}(z) J_{l}(k r) \cos l \theta \\
& \psi_{m}(r, \theta, z ; k)=\psi_{m}(z) J_{l}(k r) \cos l \theta \\
& \chi_{m}(r, \theta, z ; k)=\chi_{m}(z) J_{l}(k r) \sin l \theta
\end{aligned}
$$

we substitute relations (5) and (6) into equations (4). Equating integrands, we obtain

$$
\begin{aligned}
& q_{m}(r, \theta, z ; k)=\left\{\left[\varphi_{m}(z)+\frac{d \psi_{m}(z)}{d z}\right] \frac{d J_{l}(k r)}{d r}+\chi_{m}(z) \frac{J_{l}(k r)}{r}\right\} \cos l \theta \\
& \equiv\left\{\frac{1}{k} \frac{\dot{u}_{R_{m}}(z)}{c} \frac{d J_{l}(k r)}{d k r}-\frac{i}{k} \frac{\dot{v}_{L_{m}}(z)}{c} \frac{J_{l}(k r)}{k r}\right\} \cos l \theta \\
& v_{m}(r, \theta, z ; k)=\left\{-\left[\varphi_{m}(z)+\frac{d \psi_{m}(z)}{d z}\right] \frac{J_{l}(k r)}{r}-\chi_{m}(z) \frac{d J_{l}(k r)}{d r}\right\} \sin l \theta \\
& \equiv\left\{-\frac{1}{k} \frac{\dot{u}_{R_{m}}(z)}{c} \frac{J_{l}(k r)}{k r}+\frac{i}{k} \frac{\dot{v}_{L_{m}}(z)}{c} \frac{d J_{l}(k r)}{d k r}\right\} \sin l \theta \\
& w_{m}(r, \theta, z ; k)=\left[\frac{d \bar{\varphi}_{m}(z)}{d z}+\frac{d^{2} \psi_{m}(z)}{d z^{2}}+k_{\beta_{m}}^{2} \psi_{m}(z)\right] J_{l}(k r) \cos l \theta \\
& \equiv-\frac{i}{k} \frac{\dot{w}_{R_{m}}(z)}{c} J_{l}(k r) \cos l \theta \\
& P_{z z_{m}}(r, \theta, z ; k)=\left\{2 \mu_{m}\left[\frac{d^{2} \varphi_{m}(z)}{d z^{2}}+\frac{d^{3} \psi_{m}(z)}{d z^{3}}+k_{\beta_{m}}^{2} \frac{d \psi_{m}(z)}{d z}\right]-\lambda_{m} k_{\alpha_{m}}^{2} \varphi_{m}(z)\right\} \\
& \cdot J_{l}(k r) \cos l \theta \equiv \sigma_{R_{m}}(z) J_{l}(k r) \cos l \theta \\
& P_{\theta z_{m}}(r, \theta, z ; k)=-\mu_{m}\left\{\left[2 \frac{d \bar{\varphi}_{m}(z)}{d z}+2 \frac{d^{2} \psi_{m}(z)}{d z^{2}}+k_{\beta_{m}}^{2} \psi_{m}(z)\right] \frac{J_{l}(k r)}{r}\right. \\
& \left.+\frac{d \chi_{m}(z)}{d z} \frac{d J_{l}(k r)}{d r}\right\} \sin l \theta \equiv\left\{-i \tau_{R_{m}}(z) \frac{J_{l}(k r)}{k r}-\tau_{L_{m}}(z) \frac{d J_{l}(k r)}{d k r}\right\} \sin l \theta \\
& P_{r s_{m}}(r, \theta, z ; k)=\mu_{m}\left\{\left[2 \frac{d \varphi_{m}(z)}{d z}+2 \frac{d^{2} \psi_{m}(z)}{d z^{2}}+k_{\beta_{m}}^{2} \psi_{m}(z)\right] \frac{d J_{l}(k r)}{d k r}\right. \\
& \left.+\frac{d \chi_{m}(z)}{d z} \frac{J_{l}(k r)}{r}\right\} \cos l \theta \equiv\left\{i \tau_{R_{m}}(z) \frac{d J_{l}(k r)}{d k r}+\tau_{L_{m}}(z) \frac{J_{l}(k r)}{k r}\right\} \cos l \theta
\end{aligned}
$$


where we have used the following relations:

$$
\begin{aligned}
\frac{d^{2} \varphi_{m}(z)}{d z^{2}} & =\left(k^{2}-k_{\alpha_{m}}^{2}\right) \varphi_{m}(z) \equiv-k^{2} r_{\alpha_{m}}^{2} \varphi_{m}(z) \\
\frac{d^{2} \psi_{m}(z)}{d z^{2}} & =\left(k^{2}-k_{\beta_{m}}^{2}\right) \psi_{m}(z) \equiv-k^{2} r_{\beta_{m}}^{2} \psi_{m}(z) \\
\frac{d^{2} \chi_{m}(z)}{d z^{2}} & =\left(k^{2}-k_{\beta_{m}}^{2}\right) \chi_{m}(z) \equiv-k^{2} r_{\beta_{m}}^{2} \chi_{m}(z) \\
c & =\frac{\omega}{k}
\end{aligned}
$$

the first three relations ( 8 ) being a consequence of substituting equations (6) and (5) into equations (3).

Since there are no boundaries at $r$ or $\theta$ constant, the solution to the multilayered half-space problem will have the same $r$ and $\theta$ dependence as the source integrands. The $r$ and $\theta$ dependence of equation (6) were chosen for this reason. For the horizontal point force their dependence is given by $l=1$ and for the azimuthal independent sources by $l=0$.

At the welded interface between two layers, we impose the boundary condition of continuity of displacement and stress. Since $\left(J_{l}(k r)\right) / k r$ and $\left(d J_{l}(k r)\right) / d k r$ are linearly independent, we must impose continuity on their individual coefficients in the integrand expressions (7) in order that continuity of displacement and stress be satisfied for all $r$. From equations $(7)$ this continuity at the $z=z_{m-1}$ interface between layers $m$ and $m-1$ can be expressed in vector form as follows.

$$
\left[\begin{array}{l}
\frac{\dot{u}_{R_{m}}\left(z_{m-1}\right)}{c} \\
\frac{\dot{w}_{R_{m}}\left(z_{m-1}\right)}{c} \\
\frac{\sigma_{R_{m}}\left(z_{m-1}\right)}{c} \\
\tau_{R_{m}}\left(z_{m-1}\right)
\end{array}\right]=\left[\begin{array}{l}
\frac{\dot{u}_{R_{m-1}}\left(z_{m-1}\right)}{c} \\
\frac{\dot{w}_{R_{m-1}}\left(z_{m-1}\right)}{c} \\
\frac{\sigma_{R_{m-1}}\left(z_{m-1}\right)}{c} \\
\tau_{R_{m-1}}\left(z_{m-1}\right)
\end{array}\right]
$$

and

$$
\left[\begin{array}{c}
\frac{\dot{v}_{I_{m}}\left(z_{m-1}\right)}{c} \\
\tau_{L_{m}}\left(z_{m-1}\right)
\end{array}\right]=\left[\begin{array}{l}
\frac{\dot{v}_{L_{m-1}}\left(z_{m-1}\right)}{c} \\
\tau_{L_{m-1}}\left(z_{m-1}\right)
\end{array}\right]
$$

From (7) we see that these $z$ dependent coefficients of the displacement and stress integrands for layer $m$ are given by 
634 BULLETIN OF THE SEISMOLOGICAL SOCIETY OF AMERICA.

$$
\begin{aligned}
& \frac{\dot{u}_{R_{m}}(z)}{c}=k^{2}\left[\varphi_{m}(z)+\frac{d \psi_{m}(z)}{d z}\right] \\
& \frac{\dot{w}_{R_{m}}(z)}{c}=i k\left[\frac{d \varphi_{m}(z)}{c z}+\frac{d^{2} \psi_{m}(z)}{d z^{2}}+k_{\beta_{m}}^{2} \psi_{m}(z)\right] \\
& \sigma_{R_{m}}(z)=2 \mu_{m}\left[\frac{d^{2} \varphi_{m}(z)}{d z^{2}}+\frac{d^{3} \psi_{m}(z)}{d z^{3}}+k_{\beta_{m}}^{2} \frac{d \psi_{m}(z)}{d z}\right]-\lambda_{m} k_{\alpha_{m} \varphi_{m}}^{2}(z) \\
& \tau_{R_{m}}(z)=-i k_{\mu_{m}}\left[2 \frac{d \varphi_{m}(z)}{d z}+2 \frac{d^{2} \psi_{m}(z)}{d z^{2}}+k_{\beta_{m}}^{2} \psi_{m}(z)\right]
\end{aligned}
$$

and

$$
\begin{aligned}
& \frac{\dot{v}_{L_{m}}(z)}{c}=i k^{2} \chi_{m}(z) \\
& \tau_{L_{m}}(z)=k \mu_{m} \frac{d \chi_{m}(z)}{d z}
\end{aligned}
$$

For layers not containing a source, we use for $\varphi_{m}(z)$ and $\psi_{m}(z)$ the general solutions of equations (8) with arbitrary coefficients.

$$
\begin{aligned}
& \varphi_{m}(z)=\tilde{\Delta}_{m}{ }^{\prime} e^{-i k r_{\alpha_{m}} z}+\tilde{\Delta}_{m}{ }^{\prime \prime} e^{i k r_{\alpha_{m}} z} \\
& \psi_{m}(z)=\tilde{\omega}_{m}{ }^{\prime} e^{-i k r_{m} z}+\tilde{\omega}_{m}{ }^{\prime \prime} e^{i k \beta_{m}} z
\end{aligned}
$$

And from (8), the exponents of (11) are given by

$$
\begin{aligned}
& \left(k r_{\alpha_{m}}\right)^{2}=k_{\alpha_{m}}^{2}-k^{2}=k^{2}\left[\frac{c^{2}}{\alpha_{m}^{2}}-1\right] \\
& \left(k r_{\beta_{m}}\right)^{2}=k_{\beta_{m}}^{2}-k^{2}=k^{2}\left[\frac{c^{2}}{\beta_{m}{ }^{2}}-1\right]
\end{aligned}
$$

where $k_{\alpha_{m}}=\omega / \alpha_{m}, k_{\beta_{m}}=\omega / \beta_{m}$ and $\alpha_{m}$ and $\beta_{m}$ are the compressional and shear velocities respectively of layer $m$. We use the following sign criteria for $\gamma_{\alpha_{m}}$ and $r_{\beta_{m}}$ as given in Haskell (1953):

$$
\begin{array}{ll}
r_{\alpha_{m}}=\left[\frac{c^{2}}{\alpha_{m}^{2}}-1\right]^{1 / 2} & \text { for } c>\alpha_{m} \\
r_{\alpha_{m}}=-i\left[1-\frac{c^{2}}{\alpha_{m}^{2}}\right]^{1 / 2} & \text { for } c<\alpha_{m} \\
r_{\beta_{m}}=\left[\frac{c^{2}}{\beta_{m}^{2}}-1\right]^{1 / 2} & \text { for } c>\beta_{m} \\
r_{\beta_{m}}=-i\left[1-\frac{c^{2}}{\beta_{m}^{2}}\right]^{1 / 2} & \text { for } c<\beta_{m}
\end{array}
$$


Defining

$$
\begin{aligned}
& \hat{\Delta}_{m}{ }^{\prime}=-k^{2}\left(\frac{c}{\alpha_{m}}\right)^{2} e^{-i k r_{\alpha_{m}} z_{m-1}} \tilde{\Delta}_{m}{ }^{\prime} \\
& \hat{\Delta}_{m}{ }^{\prime \prime}=-k^{2}\left(\frac{c}{\alpha_{m}}\right)^{2} e^{i k r_{\alpha_{m}} z_{m-1}} \tilde{\Delta}_{m}{ }^{\prime \prime} \\
& \hat{\omega}_{m}{ }^{\prime}=\frac{i k^{3}}{\gamma_{m}} e^{-i k r_{\beta_{m}} z_{m-1}} \tilde{\omega}_{m}{ }^{\prime}
\end{aligned}
$$

and

$$
\hat{\omega}_{m}^{\prime \prime}=\frac{i k^{3}}{\gamma_{m}} e^{i k r_{\beta_{m}}{ }^{m-1}-1} \tilde{\omega}_{m}^{\prime \prime}
$$

and substituting equations (11) in equations (10a), we obtain

$$
\begin{aligned}
\frac{\dot{u}_{R_{m}}(z)}{c}=-\left(\frac{\alpha_{m}}{c}\right)^{2}\left[\left(\hat{\Delta}_{m}{ }^{\prime}\right.\right. & \left.+\hat{\Delta}_{m}{ }^{\prime \prime}\right) \cos k r_{\alpha_{m}}\left(z-z_{m-1}\right) \\
& \left.-i\left(\hat{\Delta}_{m}{ }^{\prime}-\hat{\Delta}_{m}{ }^{\prime \prime}\right) \sin k r_{\alpha_{m}}\left(z-z_{m-1}\right)\right] \\
& -\gamma_{m} r_{\beta_{m}}\left[\left(\hat{\omega}_{m}{ }^{\prime}-\hat{\omega}_{m}{ }^{\prime \prime}\right) \cos k r_{\beta_{m}}\left(z-z_{m-1}\right)\right. \\
& \left.-i\left(\hat{\omega}_{m}{ }^{\prime}+\hat{\omega}_{m}{ }^{\prime \prime}\right) \sin k r_{\beta_{m}}\left(z-z_{m-1}\right)\right] \\
\frac{\dot{w}_{R_{m}}(z)}{c}=-\left(\frac{\alpha_{m}}{c}\right)^{2} r_{\alpha_{m}}[ & -i\left(\hat{\Delta}_{m}{ }^{\prime}+\hat{\Delta}_{m}{ }^{\prime \prime}\right) \sin k r_{\alpha_{m}}\left(z-\hat{z}_{m-1}\right) \\
& \left.+\left(\hat{\Delta}_{m}{ }^{\prime}-\hat{\Delta}_{m}{ }^{\prime \prime}\right) \cos k r_{\alpha_{m}}\left(z-z_{m-1}\right)\right] \\
& +\gamma_{m}\left[-i\left(\hat{\omega}_{m}{ }^{\prime}-\hat{\omega}_{m}{ }^{\prime \prime}\right) \sin k r_{\beta_{m}}\left(z-z_{m-1}\right)\right. \\
& \left.+\left(\hat{\omega}_{m}{ }^{\prime}+\hat{\omega}_{m}{ }^{\prime \prime}\right) \cos k r_{\beta_{m}}\left(z-z_{m-1}\right)\right]
\end{aligned}
$$

$\sigma_{R_{m}}(z)=-\rho_{m} \alpha_{m}{ }^{2}\left(\gamma_{m-1}\right)\left[\left(\hat{\Delta}_{m}{ }^{\prime}+\hat{\Delta}_{m}{ }^{\prime \prime}\right) \cos k r_{\alpha_{m}}\left(z-z_{m-1}\right)\right.$

$$
\begin{aligned}
& \left.-i\left(\hat{\Delta}_{m}{ }^{\prime}-\hat{\Delta}_{m}{ }^{\prime \prime}\right) \sin k r_{\alpha_{m}}\left(z-z_{m-1}\right)\right] \\
& -\rho_{m} c^{2} \gamma_{m}{ }^{2} r_{\beta_{m}}\left[\left(\hat{\omega}_{m}{ }^{\prime}-\hat{\omega}_{m}{ }^{\prime \prime}\right) \cos k r_{\beta_{m}}\left(z-z_{m-1}\right)\right. \\
& \left.-i\left(\hat{\omega}_{m}{ }^{\prime}+\hat{\omega}_{m}^{\prime \prime}\right) \sin k r_{\beta_{m}}\left(z-z_{m-1}\right)\right]
\end{aligned}
$$

$\tau_{R_{m}}(z)=\rho_{m} \alpha_{m}{ }^{2} \gamma_{m} r_{\alpha_{m}}\left[-i\left(\hat{\Delta}_{m}{ }^{\prime}+\hat{\Delta}_{m}{ }^{\prime \prime}\right) \sin k r_{\alpha_{m}}\left(z-z_{m-1}\right)\right.$

$$
\begin{aligned}
& \left.+\left(\hat{\Delta}_{m}{ }^{\prime}-\hat{\Delta}_{m}{ }^{\prime \prime}\right) \cos k r_{\alpha_{m}}\left(z-z_{m-1}\right)\right] \\
& \quad-\rho_{m} c^{2} \gamma_{m}\left(\gamma_{m}-1\right)\left[-i\left(\hat{\omega}_{m}{ }^{\prime}-\hat{\omega}_{m}{ }^{\prime \prime}\right) \sin k r_{\beta_{m}}\left(z-z_{m-1}\right)\right. \\
& \left.\quad+\left(\hat{\omega}_{m}{ }^{\prime}+\hat{\omega}_{m}{ }^{\prime \prime}\right) \cos k r_{\beta_{m}}\left(z-z_{m-1}\right)\right]
\end{aligned}
$$


where we have made use of the following relations

$$
\begin{aligned}
& k_{\alpha_{m}}^{2}=\frac{k^{2} c^{2}}{\alpha_{m}^{2}}=k^{2} c^{2} \frac{\rho_{m}}{\lambda_{m}+2 \mu_{m}} \\
& k_{\beta_{m}}^{2}=\frac{k^{2} c^{2}}{\beta_{m}^{2}}=k^{2} c^{2} \frac{\rho_{m}}{\mu_{m}}
\end{aligned}
$$

and

$$
\gamma_{m}=2\left(\frac{\beta_{m}}{c}\right)^{2}
$$

Evaluating equations (14) at $z=z_{m}$ and at $z=z_{m-1}$, we can then eliminate the coefficients $\left(\hat{\Delta}_{m}{ }^{\prime}+\hat{\Delta}_{m}{ }^{\prime \prime}\right),\left(\hat{\Delta}_{m}{ }^{\prime}-\hat{\Delta}_{m}{ }^{\prime \prime}\right),\left(\hat{\omega}_{m}{ }^{\prime}-\hat{\omega}_{m}{ }^{\prime \prime}\right)$, and $\left(\hat{\omega}_{m}{ }^{\prime}+\hat{\omega}_{m}{ }^{\prime \prime}\right)$ in the same manner as Haskell (1953) and obtain his matrix result.

$$
\left[\begin{array}{c}
\frac{\dot{u}_{R_{m}}\left(z_{m}\right)}{c} \\
\frac{\dot{w}_{R_{m}}\left(z_{m}\right)}{c} \\
\sigma_{R_{m}}\left(z_{m}\right) \\
\tau_{R_{m}}\left(z_{m}\right)
\end{array}\right]=a_{R_{m}}\left[\begin{array}{c}
\frac{\dot{u}_{R_{m}}\left(z_{m-1}\right)}{c} \\
\frac{\dot{w}_{R_{m}}\left(z_{m-1}\right)}{c} \\
\sigma_{R_{m}}\left(z_{m-1}\right) \\
\tau_{R_{m}}\left(z_{m-1}\right)
\end{array}\right]
$$

The lements of the layer matrix $Q_{\mathfrak{R}_{m}}$ are given by

$$
\begin{aligned}
& \left(Q_{R_{m}}\right)_{11}=\left(Q_{R_{m}}\right)_{44}=\gamma_{m} \cos P_{m}-\left(\gamma_{m}-1\right) \cos Q_{m} \\
& \left(\mathfrak{Q}_{R_{m}}\right)_{12}=\left(\mathfrak{Q}_{R_{m}}\right)_{34}=i\left[\left(\gamma_{m}-1\right) \frac{\sin P_{m}}{r_{\alpha_{m}}}-\gamma_{m} r_{\beta_{m}} \sin Q_{m}\right] \\
& \left(Q_{R_{m}}\right)_{13}=\left(Q_{R_{m}}\right)_{24}=-\left(\rho_{m} c^{2}\right)^{-1}\left[\cos P_{m}-\cos Q_{m}\right] \\
& \left(Q_{R_{m}}\right)_{14}=i\left(\rho_{m} c^{2}\right)^{-1}\left[\frac{\sin P_{m}}{r_{\alpha_{m}}}+r_{\beta_{m}} \sin Q_{m}\right] \\
& \left(Q_{R_{m}}\right)_{21}=\left(\mathbb{Q}_{R_{m}}\right)_{43}=-i\left[\gamma_{m} r_{\alpha_{m}} \sin P_{m}+\left(\gamma_{m}-1\right) \frac{\sin Q_{m}}{\gamma_{\beta m}}\right] \\
& \left(Q_{R_{m}}\right)_{22}=\left(Q_{R_{m}}\right)_{33}=-\left(\gamma_{m}-1\right) \cos P_{m}+\gamma_{m} \cos Q_{m} \\
& \left(\widehat{Q}_{R_{m}}\right)_{2 \mathbf{3}}=i\left(\rho_{m} c^{2}\right)^{-1}\left[r_{\alpha_{m}} \sin P_{m}+\frac{\sin Q_{m}}{r_{\beta_{m}}}\right] \\
& \left(\mathfrak{Q}_{R_{m}}\right)_{31}=\left(\mathfrak{Q}_{R_{m}}\right)_{42}=\rho_{m} c^{2} \gamma_{m}\left(\gamma_{m}-1\right)\left[\cos P_{m}-\cos Q_{m}\right] \\
& \left(a_{R_{m}}\right)_{32}=i \rho_{m} c^{2}\left[\left(\gamma_{m}-1\right)^{2} \frac{\sin P_{m}}{r_{\alpha_{m}}}+{\gamma_{m}}^{2} r_{\beta_{m}} \sin Q_{m}\right] \\
& \left(\mathfrak{Q}_{R_{m}}\right)_{41}=i \rho_{m} c^{2}\left[\gamma_{m}{ }^{2} r_{\alpha_{m}} \sin P_{m}+\left(\gamma_{m}-1\right)^{2} \frac{\sin Q_{m}}{r_{\beta_{m}}}\right]
\end{aligned}
$$


with

$$
P_{m}=r k_{\alpha_{m}} d_{m}, \quad Q_{m}=k r_{\beta_{m}} d_{m} \text { and } \quad d_{m}=z_{m}-z_{m-1}
$$

In addition, the coefficients are related to the $z$ dependent quantities at $z=z_{m \rightarrow 1}$ by

$$
\left[\begin{array}{l}
\hat{\Delta}_{m}^{\prime}+\hat{\Delta}_{m}^{\prime \prime} \\
\hat{\Delta}_{m}^{\prime}-\hat{\Delta}_{m}^{\prime \prime} \\
\hat{\omega}_{m}^{\prime}-\hat{\omega}_{m}^{\prime \prime} \\
\hat{\omega}_{m}^{\prime}+\hat{\omega}_{m}^{\prime \prime}
\end{array}\right]=E_{R_{m}}^{-1}\left[\begin{array}{c}
\frac{\dot{u}_{R_{m}}\left(z_{m-1}\right)}{c} \\
\frac{\dot{w}_{R_{m}}\left(z_{m-1}\right)}{c} \\
\sigma_{R_{m}}\left(z_{m-1}\right) \\
\tau_{R_{m}}\left(z_{m-1}\right)
\end{array}\right]
$$

where

$$
E_{R_{m}}^{-1}=\left[\begin{array}{cccc}
-2\left(\frac{\beta_{m}}{\alpha_{m}}\right)^{2} & 0 & \left(\rho_{m} \alpha_{m}{ }^{2}\right)^{-1} & 0 \\
0 & c^{2}\left(\gamma_{m}-1\right) /\left(\alpha_{m}{ }^{2} \gamma_{\alpha_{m}}\right) & 0 & \left(\rho_{m} \alpha_{m}{ }^{2} r_{\alpha_{m}}\right)^{-1} \\
\left(\gamma_{m}-1\right) /\left(\gamma_{m} r_{\beta_{m}}\right) & 0 & -\left(\rho_{m} c^{2} \gamma_{m} r_{\beta_{m}}\right)^{-1} & 0 \\
0 & 1 & 0 & \left(\rho_{m} c^{2} \gamma_{m}\right)^{-1}
\end{array}\right]
$$

Equation (17) was used in obtaining (15) and it will be used again for the halfspace boundary condition.

As pointed out by Haskell (1962), the inverse of the layer matrix is given by

$$
\left(\mathfrak{Q}_{R_{m}}^{-1}\right)_{j k}=(-1)^{j+k}\left(\mathfrak{Q}_{R_{m}}\right)_{j k}
$$

Combining (19) with the relations between elements given in (16), we can write the inverse matrix as

$$
\hat{Q}_{R_{m}}^{-1}=\left[\begin{array}{rrrr}
\left(\mathfrak{Q}_{R_{m}}\right)_{44} & -\left(\mathfrak{Q}_{R_{m}}\right)_{34} & \left(\mathfrak{Q}_{R_{m}}\right)_{24} & -\left(\mathfrak{Q}_{R_{m}}\right)_{14} \\
-\left(\mathfrak{Q}_{R_{m}}\right)_{43} & \left(\mathfrak{Q}_{R_{m}}\right)_{33} & -\left(\mathfrak{Q}_{R_{m}}\right)_{23} & \left(\mathfrak{Q}_{R_{m}}\right)_{13} \\
\left(\mathfrak{Q}_{R_{m}}\right)_{42} & -\left(\mathfrak{Q}_{R_{m}}\right)_{32} & \left(\mathfrak{Q}_{R_{m}}\right)_{22} & -\left(\mathfrak{Q}_{R_{m}}\right)_{12} \\
-\left(\mathfrak{Q}_{R_{m}}\right)_{41} & \left(\mathfrak{Q}_{R_{m}}\right)_{31} & \left.-) \mathfrak{Q}_{R_{m}}\right)_{21} & \left(\mathfrak{Q}_{R_{m}}\right)_{11}
\end{array}\right]
$$

In a similar manner, we form $\chi_{m}(z)$ from the general solutions of $(8)$ for layers without a source

$$
\chi_{m}(z)=\tilde{\boldsymbol{\epsilon}}_{m}{ }^{\prime} e^{-i k r_{\beta m} z}+\tilde{\boldsymbol{\epsilon}}_{m}{ }^{\prime \prime} e^{i k r_{\beta m} z}
$$

and substitute into equations $(9 \mathrm{~b})$. Defining 


$$
\hat{\boldsymbol{\epsilon}}_{m}{ }^{\prime}=k e^{-i k r \beta_{m} z_{m-1}} \tilde{\boldsymbol{\epsilon}}_{m}{ }^{\prime} \text { and } \hat{\boldsymbol{\epsilon}}_{m}{ }^{\prime \prime}=k e^{i k r_{\beta m} z_{m-1}} \tilde{\boldsymbol{\epsilon}}_{m}{ }^{\prime \prime}
$$

we obtain

$$
\begin{aligned}
\begin{aligned}
& \frac{\dot{v}_{L_{m}}(z)}{c}=\left(\hat{\epsilon}_{m}{ }^{\prime}+\hat{\epsilon}_{m}{ }^{\prime \prime}\right) i k \cos k r_{\beta_{m}}\left(z-z_{m-1}\right)+\left(\hat{\epsilon}_{m}{ }^{\prime}-\hat{\epsilon}_{m}{ }^{\prime \prime}\right) k \sin k r_{\beta_{m}}\left(z-z_{m-1}\right) \\
& \tau_{L_{m}}(z)=-\left(\hat{\epsilon}_{m}{ }^{\prime}+\hat{\epsilon}_{m}{ }^{\prime \prime}\right) k \mu_{m} r_{\beta_{m}} \sin k r_{\beta_{m}}\left(z-z_{m-1}\right) \\
&-\left(\hat{\epsilon}_{m}{ }^{\prime}-\hat{\epsilon}_{m}{ }^{\prime \prime}\right) i k \mu_{m} r_{\beta_{m}} \cos k r_{\beta_{m}}\left(z-z_{m-1}\right)
\end{aligned}
\end{aligned}
$$

Evaluating equations (23) at $z=z_{m}$ and at $z=z_{m-1}$ we can eliminate the coefficients and obtain the following:

$$
\left[\begin{array}{c}
\frac{\dot{v}_{L_{m}}\left(z_{m}\right)}{c} \\
\tau_{L_{m}}\left(z_{m}\right)
\end{array}\right]=\mathfrak{Q}_{L_{m}}\left[\begin{array}{c}
\frac{\dot{v}_{L_{m}}\left(z_{m-1}\right)}{c} \\
\tau_{L_{m}}\left(z_{m-1}\right)
\end{array}\right]
$$

where the elements of $\mathscr{Q}_{L_{m}}$ are

$$
\begin{aligned}
& \left(\mathscr{Q}_{L_{m}}\right)_{11}=\left(\mathfrak{Q}_{L_{m}}\right)_{22}=\cos Q_{m} \\
& \left(\mathfrak{Q}_{L_{m}}\right)_{12}=\frac{i}{\mu_{m} r_{\beta m}} \sin Q_{m} \\
& \left(\mathfrak{Q}_{L_{m}}\right)_{21}=i \mu_{m} r_{\beta_{m}} \sin Q_{m}
\end{aligned}
$$

Here the coefficients are related to these $z$ dependent quantities at $z=z_{m-1}$ by

$$
\left[\begin{array}{c}
\hat{\boldsymbol{\epsilon}}_{m}{ }^{\prime}+\hat{\boldsymbol{\epsilon}}_{m}{ }^{\prime \prime} \\
\hat{\epsilon}_{m}{ }^{\prime}-\hat{\epsilon}_{m}{ }^{\prime \prime}
\end{array}\right]=E_{L_{m}}^{-1}\left[\begin{array}{c}
\frac{\dot{v}_{x_{m}}\left(z_{m-1}\right)}{c} \\
\tau_{L_{m}}\left(z_{m-1}\right)
\end{array}\right]
$$

where

$$
E_{L_{m}}^{-1}=\left[\begin{array}{cc}
(i k)^{-1} & 0 \\
0 & -\left(i k \mu_{m} r_{\beta_{m}}\right)^{-1}
\end{array}\right]
$$

The inverse of the layer matrix $a_{L_{m}}$ is given by

$$
Q_{L_{m}}^{-1}=\left[\begin{array}{cc}
\left(Q_{L_{m}}\right)_{22} & -\left(Q_{L_{m}}\right)_{12} \\
-\left(Q_{L_{m}}\right)_{21} & \left(Q_{L_{m}}\right)_{11}
\end{array}\right]
$$


Comparing equation (28) with (20) we see that the elements of the inverse of both $\mathfrak{Q}_{R_{m}}$ and $\mathfrak{Q}_{L_{m}}$ can be written as

$$
\left(a^{-1}\right)_{j k}=(-1)^{j+k}(a)_{l_{p}}
$$

where $l=n+1-k, p=n+1-j$ and $a$ is a $n \times n$ matrix. It is easy to show that if

$$
c=a b
$$

and the inverse of matrices $a$ and $b$ are given by relation (29) then the inverse of the product matrix $c$ will also be given by relation (29). This is an important matrix relation which will be used later to simplify the form of our solution.

\section{Matrix Relations for the Source Layer}

The point sources considered in this paper can be represented in two equivalent forms. The first form is the source potential. For the source layer $s$ defined by the planes $z_{s}$ and $z_{s-1}$, we use a general point source located at $D,\left(z_{s}<D<z_{s-1}\right)$ such that the $z$-dependent integrands of the source potentials are solutions of equations (8) everywhere in $s$ and continuous with continuous derivatives except at $D$ or

and

$$
\begin{array}{ll}
\varphi_{s 0}(z)=S_{01}^{ \pm} e^{-i k r_{\alpha_{s}}|z-D|} & \\
\psi_{s 0}(z)=S_{02}^{ \pm} e^{-i k r_{s}|z \sim D|} & \pm \text { as } z \gtrless D
\end{array}
$$

$$
\chi_{80}(z)=S_{03}^{ \pm} e^{-i k r_{\beta}|z-D|}
$$

where $S_{01}^{ \pm}, S_{02}^{ \pm}$and $S_{03}^{ \pm}$are spatially independent constants which depend on the source type and the elastic constants of the layer containing the source.

The second form is a discontinuity in the $z$ dependent coefficients of the displacement and stress integrands at the source plane. The equivalence of the two forms will become obvious as we obtain the necessary matrix relations for the source layer.

Combining equations (30) with the solutions of equations (8) with arbitrary coefficients, the general potentials for the source layer are

$$
\begin{aligned}
& \varphi_{s}(z)=S_{01}^{ \pm} e^{-i k r_{\alpha_{s}}|z-D|}+\tilde{\Delta}_{s}^{\prime} e^{-i k r_{\alpha_{s}} z}+\tilde{\Delta}_{s}^{\prime \prime} e^{i k r_{\alpha_{s}} z} \\
& \psi_{s}(z)=S_{02}^{ \pm} e^{-i k r \beta_{s}|z-D|}+\tilde{\omega}_{s}^{\prime} e^{-i k r_{s} z}+\bar{\omega}_{s}^{\prime \prime} e^{i k r_{\beta_{s}} z} \\
& \chi_{s}(z)=S_{03}^{ \pm} e^{-i k r_{\beta_{s}}|z-D|}+\tilde{\boldsymbol{\epsilon}}_{s}^{\prime} e^{-i k r_{\beta_{s}} z}+\tilde{\epsilon}_{s}^{\prime \prime} e^{i k r_{\beta_{s}} z}
\end{aligned}
$$

or rewriting 


$$
\begin{aligned}
& \varphi_{s}(z)=\left(S_{01}^{+} e^{i k r_{\alpha_{s}} D}+\tilde{\Delta}_{s}^{\prime}\right) e^{-i k r_{\alpha_{s}} z}+\tilde{\Delta}_{s}^{\prime \prime} e^{i k r_{\alpha_{s}} z} \\
& \psi_{s}(z)=\left(S_{02}^{+} e^{i k r_{\beta_{s}} D}+\tilde{\omega}_{s}^{\prime}\right) e^{-i k r_{\beta_{s}} z}+\tilde{\omega}_{s}^{\prime \prime} e^{i k r_{\beta_{s}} z} \quad z_{s} \geqq z>D \\
& \chi_{s}(z)=\left(S_{03}^{+} e^{i k r_{\beta_{s}} D}+\tilde{\epsilon}_{s}^{\prime}\right) e^{-i k r \beta_{s} z}+\tilde{\epsilon}_{s}^{\prime \prime} e^{i k r_{s} z} \\
& \varphi_{s}(z)=\tilde{\Delta}_{s}^{\prime} e^{-i k r_{s} z}+\left(\tilde{\Delta}_{s}^{\prime \prime}+S_{10}^{-} e^{-i k r_{\alpha_{s}} D}\right) e^{i k r_{\alpha_{s}} z} \quad D>z \geqq z_{s-1} \\
& \psi_{s}(z)=\tilde{\omega}_{s}^{\prime} e^{-i k r_{s} z}+\left(\tilde{\omega}_{s}^{\prime \prime}+S_{02}^{-} e^{-i k r_{s} D}\right) e^{i k r_{s} z} \\
& \chi_{s}(z)=\tilde{\epsilon}_{s}^{\prime} e^{-i k r \beta_{s} z}+\left(\tilde{\epsilon}_{s}^{\prime \prime}+S_{03}^{-} e^{-i k r_{\beta_{s}} D}\right) e^{i k r_{\beta_{s}} z}
\end{aligned}
$$

Decomposing layer $s$ into two layers with the same elastic constants; layer $s_{2}$ for $z_{s} \geqq z \geqq D$ and layer $s_{1}$ for $D \geqq z \geqq z_{s-1}$, and defining new constants

$$
\begin{array}{ll}
\tilde{\Delta}_{s 2}^{\prime}=S_{01}^{+} e^{i k r_{\alpha_{s}} D}+\tilde{\Delta}_{s}^{\prime}, & \tilde{\Delta}_{s 2}^{\prime \prime}=\tilde{\Delta}_{s}^{\prime \prime} \\
\tilde{\omega}_{s 2}^{\prime}=S_{02}^{+} e^{i k r_{s} D}+\tilde{\omega}_{s}^{\prime}, & \tilde{\omega}_{s 2}^{\prime \prime}=\bar{\omega}_{s}^{\prime \prime} \\
\tilde{\epsilon}_{s 2}^{\prime}=S_{03}^{+} e^{i k r_{\beta_{s}} D}+\tilde{\epsilon}_{s}^{\prime}, & \tilde{\epsilon}_{s 2}^{\prime \prime}=\tilde{\epsilon}_{s}^{\prime \prime} \\
\tilde{\Delta}_{s 1}^{\prime}=\tilde{\Delta}_{s}^{\prime}, & \tilde{\Delta}_{s 1}^{\prime \prime}=\tilde{\Delta}_{s}^{\prime \prime}+S_{01}^{-} e^{-i k r_{\alpha_{s}} D} \\
\tilde{\omega}_{s 1}^{\prime}=\tilde{\omega}_{s}^{\prime}, & \tilde{\omega}_{s 1}^{\prime \prime}=\tilde{\omega}_{s}^{\prime \prime}+S_{02}^{-} e^{-i k r_{s} D} \\
\tilde{\epsilon}_{s 1}^{\prime}=\tilde{\epsilon}_{s}^{\prime}, \quad \text { and } & \tilde{\epsilon}_{s 1}^{\prime \prime}=\tilde{\epsilon}_{s}^{\prime \prime}+S_{03}^{-} e^{-i k r_{\beta_{s}} D}
\end{array}
$$

we can write equation (32) as

$$
\begin{aligned}
& \varphi_{s 2}(z)=\tilde{\Delta}_{s 2}^{\prime} e^{-i k r_{\alpha_{s}} z}+\tilde{\Delta}_{s 2}^{\prime \prime} e^{i k r_{\alpha_{s}} z} \\
& \psi_{s 2}(z)=\tilde{\omega}_{s 2}^{\prime} e^{-i k r_{s} z}+\tilde{\omega}_{s 2}^{\prime \prime} e^{-i k r_{s} z} \quad z_{s} \geqq z \geqq D \\
& \chi_{s 2}(z)=\tilde{\epsilon}_{s 2}^{\prime} e^{-i k r_{\beta_{s}} z}+\tilde{\epsilon}_{s 2}^{\prime \prime} e^{i k r_{s} z} \\
& \varphi_{s 1}(z)=\tilde{\Delta}_{s 1}^{\prime} e^{-i k r_{\alpha_{s}} z}+\tilde{\Delta}_{s 1}^{\prime \prime} e^{i k r_{\alpha_{s}} z} \\
& \psi_{s 1}(z)=\tilde{\omega}_{s 1}^{\prime} e^{-i k r_{s} z}+\tilde{\omega}_{s 1}^{\prime \prime} e^{i k r \beta_{s} z} \\
& \chi_{s 1}(z)=\tilde{\epsilon}_{s 1}^{\prime} e^{-i k r_{\beta_{s}} z}+\tilde{\epsilon}_{81}^{\prime \prime} e^{i k r_{\beta_{s}} z}
\end{aligned}
$$

Comparing equations (34) with equations (11) and (21) and the relations derived from (11) and (21), we have the following matrix relations for the source layer 


$$
\begin{aligned}
& {\left[\begin{array}{c}
\frac{\dot{u}_{R_{s 2}}\left(z_{s}\right)}{c} \\
\frac{\dot{w}_{R_{s 2}}\left(z_{2}\right)}{c} \\
\sigma_{R_{s 2}}\left(z_{s}\right) \\
\tau_{R_{s 2}}\left(z_{s}\right)
\end{array}\right]=Q_{R_{s 2}}\left[\begin{array}{c}
\frac{\dot{u}_{R_{s 2}}(D)}{c} \\
\frac{\dot{w}_{R_{s 2}}(D)}{c} \\
\sigma_{R_{s 2}}(D) \\
\tau_{R_{s 2}}(D)
\end{array}\right]} \\
& {\left[\begin{array}{c}
\frac{\dot{u}_{R_{s 1}}(D)}{c} \\
\frac{\dot{w}_{R_{s 1}}(D)}{c} \\
\sigma_{R_{s 1}}(D) \\
\tau_{R_{s 1}}(D)
\end{array}\right]=\mathbb{Q}_{R_{s 1}}\left[\begin{array}{l}
\frac{\dot{u}_{R_{s 1}}\left(z_{s-1}\right)}{c} \\
\frac{\dot{w}_{R_{s 1}}\left(z_{s-1}\right)}{c} \\
\sigma_{R_{s 1}}\left(z_{s-1}\right) \\
\tau_{R_{s 1}}\left(z_{s-1}\right)
\end{array}\right]}
\end{aligned}
$$

where the elements $\mathfrak{C}_{R_{s 2}}$ and $\mathfrak{Q}_{R_{s 1}}$ are identical with the exception that

$$
d_{s 2}=z_{s}-D \quad \text { and } \quad d_{s 1}=D-z_{s-1}
$$

Furthermore it can be shown that their matrix product yields

$$
\mathfrak{Q}_{R_{s}}=\mathfrak{Q}_{R_{s 2}} \mathfrak{Q}_{R_{s 1}}
$$

where $\mathfrak{Q}_{R_{\varepsilon}}$ is the layer matrix for layer $s$ if no source is present. In addition we have the vector equation

$$
\left[\begin{array}{c}
\frac{\dot{u}_{R_{s 2}}(D)}{c} \\
\frac{\dot{w}_{R_{s 2}}(D)}{c} \\
\sigma_{R_{s 2}}(D) \\
\tau_{R_{s 2}}(D)
\end{array}\right]=\left[\begin{array}{c}
\frac{\dot{u}_{R_{s 1}}(D)}{c} \\
\frac{\dot{w}_{R_{s 1}}(D)}{c} \\
\sigma_{R_{s 1}}(D) \\
\tau_{R_{81}}(D)
\end{array}\right]+\left[\begin{array}{c}
\delta\left(\frac{\dot{u}_{R_{s}}}{c}\right) \\
\delta\left(\frac{\dot{w}_{R_{s}}}{c}\right) \\
\delta \sigma_{R_{s}} \\
\delta \tau_{R_{s}}
\end{array}\right]
$$

where from equations (10a) and (32)

$$
\begin{aligned}
\delta\left(\frac{\dot{u}_{R_{s}}}{c}\right) & =k^{2}\left[\left(S_{01}^{+}-S_{01}^{-}\right)-i k r_{\beta_{s}}\left(S_{02}^{+}+S_{02}^{-}\right)\right] \\
\delta\left(\frac{\dot{w}_{R_{s}}}{c}\right) & =i k\left[-i k r_{\alpha_{s}}\left(S_{01}^{+}+S_{01}^{-}\right)+k^{2}\left(S_{02}^{+}-S_{02}^{-}\right)\right] \\
\delta \sigma_{R_{s}} & =k^{2} c^{2} \rho_{s}\left[\left(\gamma_{s}-1\right)\left(S_{01}^{+}-S_{01}^{-}\right)-i k r_{\beta_{s}} \gamma_{s}\left(S_{02}^{+}+S_{02}^{-}\right)\right] \\
\delta \tau_{R_{s}} & =k^{2} c^{2} \rho_{s}\left[-\gamma_{s} r_{\alpha_{s}}\left(S_{01}^{+}+S_{01}^{-}\right)-i k\left(\gamma_{s}-1\right)\left(S_{02}^{+}-S_{02}^{-}\right)\right]
\end{aligned}
$$


Furthermore we obtain

$$
\begin{gathered}
{\left[\begin{array}{c}
\dot{v}_{L_{s 2}}\left(z_{s}\right) \\
c \\
\tau_{L_{s 2}}\left(z_{s}\right)
\end{array}\right]=Q_{L_{s 2}}\left[\begin{array}{c}
\frac{\dot{v}_{L_{s 2}}(D)}{c} \\
\tau_{L_{s 2}}(D)
\end{array}\right]} \\
{\left[\begin{array}{c}
\frac{\dot{v}_{L_{s 1}}(D)}{c} \\
\tau_{L_{s 1}}(D)
\end{array}\right]=Q_{L_{s 1}}\left[\begin{array}{c}
\frac{\dot{v}_{L_{s 1}}\left(z_{s-1}\right)}{c} \\
\tau_{L_{s 1}}\left(z_{s-1}\right)
\end{array}\right]}
\end{gathered}
$$

and the vector equation

$$
\left[\begin{array}{c}
\dot{v}_{L_{s 2}}(D) \\
c \\
\tau_{L_{s 2}}(D)
\end{array}\right]=\left[\begin{array}{c}
\frac{\dot{v}_{L_{s 1}}(D)}{c} \\
\tau_{L_{s 1}}(D)
\end{array}\right]+\left[\begin{array}{c}
\delta\left(\frac{\dot{v}_{L_{s}}}{c}\right) \\
\delta \tau_{L_{s}}
\end{array}\right]
$$

where by equations (10b) and (32)

$$
\begin{aligned}
\delta\left(\frac{\dot{v}_{L_{s}}}{c}\right) & =i k^{2}\left(S_{03}^{+}-S_{03}^{-}\right) \\
\delta \tau_{L_{s}} & =-i k^{2} \mu_{s} \gamma_{\beta_{s}}\left(S_{03}^{+}+S_{03}^{-}\right)
\end{aligned}
$$

\section{Rayleigh Waves from an Explostye Source at Depth}

For an explosive source, we use the Fourier time transformed spherical compressional potential for a pressure applied to the walls of a spherical cavity in medium $s$. It must be pointed out that only the source term "sees" the spherical cavity. In other words, we do not impose on the multilayer problem the boundary condition that normal and tangential stress over the cavity walls vanish for the homogeneous terms; thus waves reflected and scattered by the cavity are not considered.

The potential for this type of explosive source has been given by many authors (Kawasumi and Yosiyama, 1935; Sharp, 1942; Fu, 1945; Mengel, 1951; Blake, 1952). Here we use the following form

$$
\bar{\varphi}_{s 0}(R)=-\frac{\bar{p}_{0 s}}{4 \mu_{s}} a_{s}^{3} \frac{e^{i\left(k_{\alpha_{s}} a_{s}-\theta_{S P}\right)}}{\left[\left(1-\frac{a_{s}^{2} k_{\beta_{s}}^{2}}{4}\right)^{2}+k_{\alpha_{s}}^{2} a_{s}^{2}\right]^{1 / 2}} \frac{e^{-i k_{\alpha_{s} R} R}}{R}
$$

where

$R$ is the distance from the source,

$a_{s}$ is the cavity radius,

$\bar{p}_{0 s}$ is the Fourier time transformed pressure at the cavity walls, and

$$
\theta_{S P}=\tan ^{-1} \frac{k_{\alpha_{s}} a_{s}}{\left(1-\frac{a_{s}^{2} k_{\beta_{s}}^{2}}{4}\right)}
$$


Since the source is located at $(0, \theta, D)$ in the cylindrical coordinate system $(r$, $\theta, z$ ), we can write $R$ as

$$
R=\left[r^{2}+(z-D)^{2}\right]^{1 / 2}
$$

By means of the Sommerfeld integral, we can rewrite equation (41) as

$$
\bar{\varphi}_{s 0}(r, z)=\int_{0}^{\infty} S_{01} e^{-i k r_{\alpha_{s}}|z-D|} J_{0}(k r) d k
$$

where

and as before

$$
S_{01}=i \frac{\bar{p}_{0 s} a_{s}^{3} e^{i\left(k_{\alpha_{s}} a_{s}-\theta_{S P}\right)}}{4 \mu_{s} r_{\alpha_{s}}\left[\left(1-\frac{a_{s}^{2} k_{\beta_{s}}^{2}}{4}\right)^{2}+k_{\alpha_{s}}^{2} a_{s}{ }^{2}\right]^{1 / 2}}
$$

$$
k^{2} r_{\alpha_{s}}^{2}=k_{\alpha_{s}}^{2}-k^{2}
$$

Since the source is symmetric and the only boundary conditions are at the layer interfaces, $z$ constant, the problem is axially symmetric. Therefore setting $l=0$ in relations (6) and (7), the potential, displacement, and stress integrands for a layer $m$ reduce to

$$
\begin{gathered}
\varphi_{m}(r, \theta, z ; k)=\varphi_{m}(z) J_{0}(k r) \\
\psi_{m}(r, \theta, z ; k)=\psi_{m}(z) J_{0}(k r) \\
\chi_{m}(r, \theta, z ; k) \equiv 0 \\
q_{m}(r, \theta, z ; k)=-k\left[\varphi_{m}(z)+\frac{d \psi_{m}(z)}{d z}\right] J_{1}(k r) \equiv-\frac{1}{k} \frac{\dot{u}_{R_{m}}(z)}{c} J_{1}(k r) \\
v_{m}(r, \theta, z ; k) \equiv 0 \\
w_{m}(r, \theta, y ; k)=\left[\frac{d \varphi_{m}(z)}{d z}+\frac{d^{2} \psi_{m}(z)}{d z^{2}}+k_{\beta_{m}}^{2} \psi_{m}(z)\right] J_{0}(k r) \\
P_{z z_{m}}(r, \theta, z ; k)=\left\{2 \mu_{m}\left[\frac{d^{2} \varphi_{m}(z)}{d z^{2}}+\frac{d^{3} \psi_{m}(z)}{d z^{3}}+k_{\beta_{m}}^{2} \frac{d \psi_{m}(z)}{d z}\right]\right. \\
\left.-\lambda_{m} k_{\alpha_{m}}^{2} \varphi_{m}(z)\right\} J_{0}(k r) \equiv \sigma_{R_{m}}(z) J_{0}(k r) \\
P_{\theta z_{m}}(r, \theta, z ; k) \equiv 0 \\
P_{r_{z}}(r, \theta, z ; k)=-k J_{0}(k r) \\
{\left[2 \frac{d \varphi_{m}(z)}{d z}+2 \frac{d^{2} \psi_{m}(z)}{d z^{2}}+k_{\beta_{m}}^{2} \frac{d \psi_{m}(z)}{d z}\right] J_{1}(k r)} \\
\equiv-i \tau_{R_{m}}(z) J_{1}(k r)
\end{gathered}
$$


For the source layer, $s$, the integrand of the source term is from equation (43)

$$
\varphi_{s 0}(r, \theta, z ; k)=\varphi_{s 0}(z) J_{0}(k r)
$$

where

$$
\varphi_{s 0}(z)=S_{01} e^{-i k r_{\alpha_{s}}|z-D|}
$$

Comparing equations (47) and (30) we see that $S_{01}^{+}=S_{01}^{-}=S_{01}$ and $S_{02}^{ \pm}=S_{03}^{ \pm}=0$ and thus, by equations (37) and (40)

$$
\begin{aligned}
\delta\left(\frac{\dot{u}_{R_{s}}}{c}\right) & =0 \\
\delta\left(\frac{\dot{w}_{R^{s}}}{c}\right) & =2 k^{2} r_{\alpha_{s}} S_{01} \\
\delta \sigma_{R_{s}} & =0 \\
\delta \tau_{R_{s}} & =-2 k^{2} c^{2} \rho_{s} \gamma_{s} r_{\alpha_{s}} S_{01}=-4 k^{2} \mu_{s} r_{\alpha_{s}} S_{01} \\
\delta\left(\frac{\dot{v}_{L_{s}}}{c}\right) & =0 \\
\delta \tau_{L_{s}} & =0
\end{aligned}
$$

It should be pointed out that equations $(49 \mathrm{~b})$ are the reason that $\bar{\chi}_{m}(r, \theta, z)$ and $\bar{v}_{m}(r, \theta, z)$ are equal to zero and not because of the $\theta$ independence. As a counter example, we can have a ring torque source about the vertical axis which will produce $\bar{\chi}_{m}(r, \theta, z)$ or a $\bar{v}_{m}(r, \theta, z)$ displacement alone with no $\theta$-dependence.

Using source relations (49a), the source vector equation (36) may be written as

$$
\left[\begin{array}{c}
\frac{\dot{u}_{R_{s 2}}(D)}{c} \\
\frac{\dot{w}_{R_{s 2}}(D)}{c} \\
\sigma_{R_{s 2}}(D) \\
\tau_{R_{s 2}}(D)
\end{array}\right]=\left[\begin{array}{c}
\frac{\dot{u}_{R_{s 1}}(D)}{c} \\
\frac{\dot{w}_{R_{s 1}}(D)}{c} \\
\sigma_{R_{s 1}}(D) \\
\tau_{R_{s 1}}(D)
\end{array}\right]+\left[\begin{array}{c}
0 \\
\delta\left(\frac{\dot{w}_{R_{s}}}{c}\right) \\
0 \\
\delta \tau_{R_{s}}
\end{array}\right]
$$

Combining equations (9a) and (15), we have

$$
\left[\begin{array}{l}
\frac{\dot{u}_{R_{n-1}}\left(z_{n-1}\right)}{c} \\
\frac{\dot{w}_{R_{n-1}}\left(z_{n-1}\right)}{c} \\
\sigma_{R_{n-1}}\left(z_{n-1}\right) \\
\tau_{R_{n-1}}\left(z_{n-1}\right)
\end{array}\right]=A_{R}^{s 2}\left[\begin{array}{c}
\frac{\dot{u}_{R_{s 2}}(D)}{c} \\
\frac{\dot{w}_{R_{s 2}}(D)}{c} \\
\sigma_{R_{s 2}}(D) \\
\tau_{R_{s 2}}(D)
\end{array}\right]
$$




$$
\left[\begin{array}{l}
\frac{\dot{u}_{R_{s 1}}(D)}{c} \\
\frac{\dot{w}_{R_{s 1}}(D)}{c} \\
\sigma_{R_{s 1}}(D) \\
\tau_{R_{s 1}}(D)
\end{array}\right]=A_{R_{s 1}}\left[\begin{array}{c}
\frac{\dot{u}_{R_{1}}(0)}{c} \\
\frac{\dot{w}_{R_{1}}(0)}{c} \\
\sigma_{R_{1}}(0) \\
\tau_{R_{1}}(0)
\end{array}\right]
$$

where $A_{R}^{s^{2}}=\mathfrak{Q}_{R_{n-1}} \cdots \mathfrak{Q}_{R_{s 2}}$ and $A_{R_{s 1}}=\mathfrak{Q}_{R_{s 1}} \cdots \mathfrak{Q}_{R_{1}}$. At the free surface $z=0$, we require that the stresses vanish. Thus by equation (46), equation (50) reduces to

$$
\left[\begin{array}{l}
\frac{\dot{u}_{R s 1}(D)}{c} \\
\frac{\dot{w}_{R_{s 1}}(D)}{c} \\
\sigma_{R_{s 1}}(D) \\
\tau_{R_{s 1}}(D)
\end{array}\right]=A_{R_{s 1}}\left[\begin{array}{c}
\dot{u}_{R_{0}} \\
c \\
\frac{\dot{w}_{R_{0}}}{c} \\
0 \\
0
\end{array}\right]
$$

where

$$
\frac{\dot{u}_{R_{0}}}{c} \equiv \frac{\dot{u}_{R_{1}}(0)}{c} \text { and } \quad \frac{\dot{w}_{R_{0}}}{c} \equiv \frac{\dot{w}_{R_{1}}(0)}{c}
$$

We now define $W, X, Y$ and $Z$ by the matrix operation

$$
\left[\begin{array}{l}
W \\
X \\
Y \\
Z
\end{array}\right] \equiv A_{R_{s 1}}^{-1}\left[\begin{array}{c}
\frac{\dot{u}_{R_{s 1}}(D)}{c} \\
\frac{\dot{w}_{R_{s 1}}(D)}{c} \\
\sigma_{R_{s 1}}(D) \\
\tau_{R_{s 1}}(D)
\end{array}\right]
$$

Multiplying the vector source equation (50) by $A_{R_{s 1}}^{-1}$ and using equations (54) and (53) we have

$$
\left[\begin{array}{c}
W \\
X \\
Y \\
Z
\end{array}\right]=\left[\begin{array}{c}
\frac{\dot{u}_{R_{0}}}{c} \\
\frac{\dot{w}_{R_{0}}}{c} \\
0 \\
0
\end{array}\right]+A_{R_{s 1}}^{-1}\left[\begin{array}{c}
0 \\
\delta\left(\frac{\dot{w}_{R_{s}}}{c}\right) \\
0 \\
\delta \tau_{R_{s}}
\end{array}\right]
$$


or

$$
\begin{aligned}
\frac{\dot{u}_{R_{0}}}{c} & =W-\left[\delta\left(\frac{\dot{w}_{R_{s}}}{c}\right)\left(A_{R_{s 1}}^{-1}\right)_{12}+\delta \tau_{R_{s}}\left(A_{R_{s 1}}^{-1}\right)_{14}\right] \\
\frac{\dot{w}_{R_{0}}}{c} & =X-\left[\delta\left(\frac{\dot{w}_{R_{s}}}{c}\right)\left(A_{R_{s 1}}^{-1}\right)_{22}+\delta \tau_{R_{s}}\left(A_{R_{s 1}}^{-1}\right)_{24}\right] \\
Y & =\left[\delta\left(\frac{\dot{w}_{R_{s}}}{c}\right)\left(A_{R_{s 1}}^{-1}\right)_{32}+\delta \tau_{R_{s}}\left(A_{R_{s 1}}^{-1}\right)_{34}\right] \\
Z & =\left[\delta\left(\frac{\dot{w}_{R_{s}}}{c}\right)\left(A_{R_{s 1}}^{-1}\right)_{42}+\delta \tau_{R_{s}}\left(A_{R_{s 1}}^{-1}\right)_{44}\right]
\end{aligned}
$$

From relation (29) the inverse of $A_{R_{s 1}}$ is given by

$$
A_{R_{s 1}}^{-1}=\left[\begin{array}{rrrr}
\left(A_{R_{s 1}}\right)_{44} & -\left(A_{R_{s 1}}\right)_{34} & \left(A_{R_{s 1}}\right)_{24} & -\left(A_{R_{s 1}}\right)_{14} \\
-\left(A_{R_{s 1}}\right)_{43} & \left(A_{R_{s 1}}\right)_{33} & -\left(A_{R_{s 1}}\right)_{23} & \left(A_{R_{s 1}}\right)_{13} \\
\left(A_{R_{s 1}}\right)_{42} & -\left(A_{R_{s 1}}\right)_{32} & \left(A_{R_{s 1}}\right)_{22} & -\left(A_{R_{s 1}}\right)_{12} \\
-\left(A_{R_{s 1}}\right)_{41} & \left(A_{R_{s 1}}\right)_{31} & -\left(A_{R_{s 1}}\right)_{21} & \left(A_{R_{s 1}}\right)_{11}
\end{array}\right]
$$

Replacing the $\left(A_{R_{s 1}}^{-1}\right)$ elements in equation $(56)$ by their $A_{R_{s 1}}$ equivalents, yields

$$
\begin{aligned}
\frac{\dot{u}_{R_{0}}}{c} & =W+\left[\delta\left(\frac{\dot{w}_{R_{s}}}{c}\right)\left(A_{R_{s 1}}\right)_{34}+\delta \tau_{R_{s}}\left(A_{R_{s 1}}\right)_{14}\right] \\
\frac{\dot{w}_{R_{0}}}{c} & =X-\left[\delta\left(\frac{\dot{w}_{R_{s}}}{c}\right)\left(A_{R_{s 1}}\right)_{33}+\delta \tau_{R_{s}}\left(A_{R_{s 1}}\right)_{13}\right] \\
Y & =-\left[\delta\left(\frac{\dot{w}_{R_{s}}}{c}\right)\left(A_{R_{s 1}}\right)_{32}+\delta \tau_{R_{s}}\left(A_{R_{s 1}}\right)_{12}\right] \\
Z & =\left[\delta\left(\frac{\dot{w}_{R_{s}}}{c}\right)\left(A_{R_{s 1}}\right)_{31}+\delta \tau_{R_{s}}\left(A_{R_{s 1}}\right)_{11}\right]
\end{aligned}
$$

From equation (17), we have for the half-space or layer $n$

$$
\left[\begin{array}{l}
\hat{\Delta}_{n}{ }^{\prime}+\hat{\Delta}_{n}{ }^{\prime \prime} \\
\hat{\Delta}_{n}^{\prime}-\hat{\Delta}_{n}^{\prime \prime} \\
\hat{\omega}_{n}^{\prime}-\hat{\omega}_{n}^{\prime \prime} \\
\hat{\omega}_{n}^{\prime}+\hat{\omega}_{n}^{\prime \prime}
\end{array}\right]=E_{R_{n}}^{-1}\left[\begin{array}{c}
\frac{\dot{u}_{R_{n}}\left(z_{n-1}\right)}{c} \\
\frac{\dot{w}_{R_{n}}\left(z_{n-1}\right)}{c} \\
\sigma_{R_{n}}\left(z_{n-1}\right) \\
\tau_{R_{n}}\left(z_{n-1}\right)
\end{array}\right]
$$


As a boundary condition for the half-space, we require that the coefficients $\hat{\Delta}_{n}^{\prime \prime}$ and $\hat{\omega}_{n}{ }^{\prime \prime}$ vanish. For $c$ greater than either of the halfspace body velocities, this is equivalent to requiring that there be no radiation from infinity into the wave guide due to equation (14) and the sign criteria of $r_{\alpha_{n}}$ and $r_{\beta_{n}}$. Similarly for $c$ less than either of the body velocities, this is equivalent to requiring that displacements and stress remain finite as the depth becomes infinite. With this boundary condition, equation (59) reduces to

$$
\left[\begin{array}{c}
\hat{\Delta}_{n}{ }^{\prime} \\
\hat{\Delta}_{n}{ }^{\prime} \\
\hat{\omega}_{n}{ }^{\prime} \\
\hat{\omega}_{n}{ }^{\prime}
\end{array}\right]=E_{R_{n}}^{-1}\left[\begin{array}{c}
\frac{\dot{u}_{R_{n}}\left(z_{n-1}\right)}{c} \\
\frac{\dot{w}_{R_{n}}\left(z_{n-1}\right)}{c} \\
\sigma_{R_{n}}\left(z_{n-1}\right) \\
\tau_{R_{n}}\left(z_{n-1}\right)
\end{array}\right]
$$

Defining the matrix $A_{R}$ by the matrix product

$$
A_{R}=A_{R}^{s 2} A_{R_{s 1}}=\mathfrak{a}_{R_{n-1}} \cdots \mathfrak{Q}_{R_{s 2}} \mathbb{Q}_{R_{81}} \cdots \mathfrak{Q}_{R_{1}}=\mathfrak{Q}_{R_{n-1}} \cdots \mathfrak{Q}_{R_{s}} \cdots \mathfrak{Q}_{R_{1}}
$$

since it can be shown that $\mathfrak{Q}_{R_{s}}=\mathfrak{Q}_{R_{s 2}} \mathfrak{Q}_{R_{s l}}$, and in turn defining $J$ by

$$
J=E_{R_{n}}^{-1} A_{R}
$$

we obtain from equations $(60),(51)$ and (54)

$$
\left[\begin{array}{c}
\hat{\Delta}_{n}{ }^{\prime} \\
\hat{\Delta}_{n}{ }^{\prime} \\
{\hat{\omega_{n}}}^{\prime} \\
{\hat{\omega_{n}}}^{\prime}
\end{array}\right]=J\left[\begin{array}{c}
W \\
X \\
Y \\
Z
\end{array}\right]
$$

Eliminating $\hat{\Delta}_{n}{ }^{\prime}$ from the linear equations given by equation (62) yields

$$
0=\left(J_{11}-J_{21}\right) W+\left(J_{12}-J_{22}\right) X+\left(J_{12}-J_{23}\right) Y+\left(J_{14}-J_{24}\right) Z
$$

Similarly eliminating $\hat{\omega}_{n}^{\prime}$ yields

$$
0=\left(J_{31}-J_{41}\right) W+\left(J_{32}-J_{42}\right) X+\left(J_{33}-J_{43}\right) Y+\left(J_{34}-J_{44}\right) Z
$$

Solving equations (63) and (64) for $X$ and $W$ we obtain

$$
X=\frac{[G N-L H] Y+[R N-S L] Z}{[N K-L M]}
$$




$$
W=\frac{\left[G N\left(\frac{M}{N}\right)-L H\left(\frac{K}{L}\right)\right] Y+\left[R N\left(\frac{M}{N}\right)-S L\left(\frac{K}{L}\right)\right] Z}{[N K-L M}
$$

where

$$
\begin{aligned}
& \frac{G}{L}=\frac{J_{13}-J_{23}}{J_{11}-J_{21}}, \quad \frac{H}{N}=\frac{J_{33}-J_{43}}{J_{31}-J_{41}}, \quad \frac{R}{L}=\frac{J_{14}-J_{24}}{J_{11}-J_{21}} \\
& \frac{S}{N}=\frac{J_{34}-J_{44}}{J_{31}-J_{41}}, \quad \frac{K}{L}=\frac{J_{12}-J_{22}}{J_{11}-J_{21}} \quad \text { and } \quad \frac{M}{N}=\frac{J_{32}-J_{42}}{J_{31}-J_{41}}
\end{aligned}
$$

Using the definition of $J$ and the elements of $E_{R_{n}}^{-1}$ given in equation (18), we can write the following

$$
\begin{aligned}
& L=\gamma_{n} r_{\alpha_{n}}\left(A_{R}\right)_{11}+\left(\gamma_{n}-1\right)\left(A_{R}\right)_{21}-\frac{r_{\alpha_{n}}}{\rho_{n} c^{2}}\left(A_{R}\right)_{31}+\frac{\left(A_{R}\right)_{41}}{\rho_{n} c^{2}} \\
& K=\gamma_{n} r_{\alpha_{n}}\left(A_{R}\right)_{12}+\left(\gamma_{n}-1\right)\left(A_{R}\right)_{22}-\frac{r_{\alpha_{n}}}{\rho_{n} c^{2}}\left(A_{R}\right)_{32}+\frac{\left(A_{R}\right)_{42}}{\rho_{n} c^{2}} \\
& G=\gamma_{n} r_{\alpha_{n}}\left(A_{R}\right)_{13}+\left(\gamma_{n}-1\right)\left(A_{R}\right)_{23}-\frac{r_{\alpha_{n}}}{\rho_{n} c^{2}}\left(A_{R}\right)_{33}+\frac{\left(A_{R}\right)_{43}}{\rho_{n} c^{2}} \\
& R=\gamma_{n} r_{\alpha_{n}}\left(A_{R}\right)_{14}+\left(\gamma_{n}-1\right)\left(A_{R}\right)_{24}-\frac{r_{\alpha_{n}}\left(A_{R}\right)_{34}+\frac{\left(A_{R}\right)_{44}}{\rho_{n} c^{2}}}{\rho_{n} c^{2}} \\
& N=-\left(\gamma_{n}-1\right)\left(A_{R}\right)_{11}+\gamma_{n} r_{\beta_{n}}\left(A_{R}\right)_{21}+\frac{\left(A_{R}\right)_{31}}{\rho_{n} c^{2}}+\frac{r_{\beta_{n}}}{\rho_{n} c^{2}}\left(A_{R}\right)_{41} \\
& M=-\left(\gamma_{n}-1\right)\left(A_{R}\right)_{12}+\gamma_{n} r_{\beta_{n}}\left(A_{R}\right)_{22}+\frac{\left(A_{R}\right)_{32}}{\rho_{n} c^{2}}+\frac{r_{\beta_{n}}}{\rho_{n} c^{2}}\left(A_{R}\right)_{42} \\
& H=-\left(\gamma_{n}-1\right)\left(A_{R}\right)_{13}+\gamma_{n} r_{\beta_{n}}\left(A_{R}\right)_{23}+\frac{\left(A_{R}\right)_{33}}{\rho_{n} c^{2}}+\frac{\gamma_{\beta_{n}}}{\rho_{n} c^{2}}\left(A_{R}\right)_{43} \\
& S=-\left(\gamma_{n}-1\right)\left(A_{R}\right)_{14}+\gamma_{n} r_{\beta_{n}}\left(A_{R}\right)_{24}+\frac{\left(A_{R}\right)_{34}}{\rho_{n} c^{2}}+\frac{r_{\beta_{n}}}{\rho_{n} c^{2}}\left(A_{R}\right)_{44}
\end{aligned}
$$

From definitions (68) and the relations between $A_{R}$ elements, obtained by performing the operation $A_{R} A_{R}^{-1}=I$ and replacing the $A_{R}^{-1}$ elements by their $A_{R}$ equivalents, e.g.

$$
\left(A_{R}\right)_{13}\left(A_{R}\right)_{22}+\left(A_{R}\right)_{24}\left(A_{R}\right)_{11}-\left(A_{R}\right)_{14}\left(A_{R}\right)_{21}-\left(A_{R}\right)_{23}\left(A_{R}\right)_{12}=0
$$

it can be shown that

$$
R N-S L=G M-H K
$$


Using equations (65) and (66), we have from equation (58)

$$
\frac{\dot{w}_{R_{0}}}{c}=-\frac{N_{R}{ }^{(1)} N_{R}{ }^{(3)}}{F_{R}}
$$

and

$$
\frac{\dot{u}_{R_{0}}}{c}=\frac{N_{R}{ }^{(3)} N_{R}{ }^{(4)}}{F_{R}}
$$

where

$$
\begin{aligned}
F_{R} & \equiv[N K-L M] \\
N_{R}{ }^{(1)} & \equiv[G N-L H]\left\{Y+\frac{[R N-S L]}{[G N-L H]} Z\right\} \\
N_{R}{ }^{(2)} & \equiv 1+\frac{F_{R}}{N_{R}{ }^{(1)}}\left[\delta\left(\frac{\dot{w}_{R_{s}}}{c}\right)\left(A_{R_{s}}\right)_{33}+\delta \tau_{R_{s}}\left(A_{R_{s}}\right)_{13}\right] \\
N_{R}{ }^{(3)} & \equiv\left[G N\left(\frac{M}{N}\right)-L H\left(\frac{K}{L}\right)\right]\left\{Y+\frac{\left[R N\left(\frac{M}{N}\right)-S L\left(\frac{K}{L}\right)\right]}{\left.\left[G N\left(\frac{M}{N}\right)-L H\left(\frac{K}{L}\right)\right]\right\}}\right. \\
N_{R}{ }^{(4)} & \equiv 1+\frac{F_{R}}{N_{R}^{(3)}}\left[\delta\left(\frac{\dot{w}_{R_{s}}}{c}\right)\left(A_{R_{s}}\right)_{34}+\delta \tau_{R_{s}}\left(A_{R_{s}}\right)_{14}\right]
\end{aligned}
$$

It is convenient at this point to examine equations (72) when $F_{R}$ is equal to zero. From the definition of $F_{R}$ in equations (72), we have

$$
\frac{M}{N}=\frac{K}{L}
$$

Combining equations (73) and (69) yields

$$
\frac{R N-S L}{G N-H L}=\frac{K}{L}
$$

and thus for the case of $F_{R}=0$, equations (72) reduce to

$$
\begin{aligned}
& N_{R}^{(1)}=[G N-L H]\left\{Y+\frac{K}{L} Z\right\} \\
& N_{R}^{(2)}=1 \\
& N_{R}^{(3)}=\frac{K}{L} N_{R}^{(1)}
\end{aligned}
$$


and

$$
N_{R}^{(4)}=1
$$

Thus by equations (5), (46), (70) and (71), the displacements of the free surface are given by

$$
\bar{w}_{0} \equiv \bar{w}_{1}(r, \theta, 0)=i \int_{0}^{\infty} \frac{N_{R}^{(1)} N_{R}^{(2)}}{F_{R}} J_{0}(k r) d k
$$

and

$$
\bar{q}_{0} \equiv \bar{q}_{1}(r, \theta, 0)=-\int_{0}^{\infty} \frac{1}{k} \frac{N_{R}^{(3)} N_{R}^{(4)}}{F_{R}} J_{1}(k r) d k
$$

Evaluating equations (76) and (77) for the residue contribution, we obtain for each $j$ th mode or root, $\omega$ fixed, at $F_{R}\left(\omega, k_{R_{j}}\right)=0$

$$
\begin{aligned}
& \left\{\bar{w}_{0}\right\}_{R_{j}}=\frac{\pi}{k_{R_{i}}} \frac{N_{R_{j}}^{(1)} N_{R_{j}}^{(2)}}{\left(\frac{\partial F_{R}}{\partial k}\right)_{\omega, j}} H_{0}{ }^{(2)}\left(k_{R_{j}} r\right) \\
& \left\{\bar{q}_{0}\right\}_{R_{j}}=i \frac{\pi}{k_{R_{j}}} \frac{N_{R_{j}}^{(3)} N_{R_{j}}^{(4)}}{\left(\frac{\partial \bar{F}_{R}}{\partial k}\right)_{\omega, j}} H_{1}^{(2)}\left(k_{R_{j}} r\right)
\end{aligned}
$$

or by equation (75)

$$
\begin{aligned}
\left\{\bar{w}_{0}\right\}_{R_{j}} & =\frac{\pi}{k_{R_{j}}} \frac{N_{R_{j}}^{(1)}}{\left(\frac{\partial F_{R}}{\partial k}\right)_{\omega, j}} H_{0}{ }^{(2)}\left(k_{R_{j}} r^{r}\right) \\
\left\{\bar{q}_{0}\right\}_{R_{j}} & =i \frac{K}{\bar{L}} \frac{\pi}{k_{R_{j}}} \frac{N_{R_{j}}^{(1)}}{\left(\frac{\partial F_{R}}{\partial k}\right)_{\omega, j}} H_{1}{ }^{(2)}\left(k_{R_{j}} r\right)
\end{aligned}
$$

where $\left(\partial F_{R} / k\right)_{\omega, j}, N_{R_{j}}^{(1)}, N_{R_{j}}^{(2)}, N_{R_{j}}^{(3)}$ and $N_{R_{j}}^{(4)}$ are evaluated at $\left(\omega, k_{R_{j}}\right)$ such that $F_{R}\left(\omega, k_{R}\right)=0$.

$F_{R}\left(\omega, k_{R_{j}}\right)=0$ is a form of the period equation for Rayleigh wave propagation in plane multilayered solids (Haskell, 1953; Dorman, M. Ewing, and Oliver, 1960; Dorman and Prentiss, 1960; Press, Harkrider and Seafeldt, 1961; Dorman, 1962; Harkrider and Anderson, 1962). For all real $(\omega, k)$ or $(c, k)$ the elements in the $\mathfrak{Q}_{R_{m}}$ matrix are either always real or always imaginary according to the following criteria (Haskell, 1953). 


$$
\begin{array}{ll}
\text { Real }\left(\mathfrak{Q}_{R_{m}}\right)_{j k} & \text { if } j+k \text { even integer } \\
\text { Imaginary }\left(\mathfrak{Q}_{R_{m}}\right)_{j k} & \text { if } j+k \text { odd integer }
\end{array}
$$

The same is true also for the product matrix $A_{R}$. For a phase velocity, $c$, less than or equal to the half-space shear velocity $\beta_{n}$, we see that the quantities defined in equation (68) are also real or imaginary for all real $k$. We now express the imaginary quantities as a real quantity (designated by an asterisk superscript) multiplied by $i$ or

$$
L=i L^{*}, \quad G=i G^{*}, \quad M=i M^{*}, \text { and } S=i S^{*}
$$

and thus

$$
F_{R}(\omega, k)=N K+L^{*} M^{*}
$$

which is real for all real $k$ and $c \leqq \beta_{n}$.

Taking the ratio of $\left\{\bar{q}_{0}\right\}_{R_{j}}$ to $\left\{\bar{w}_{0}\right\}_{R_{j}}$, we obtain from equation (79)

$$
\frac{\left\{\bar{q}_{0}\right\}_{R_{j}}}{\left\{\bar{w}_{0}\right\}_{R_{j}}}=i \frac{K}{L} \frac{H_{1}^{(2)}\left(k_{R_{j}} r^{r}\right)}{H_{0}^{(2)}\left(k_{R_{j}}{ }^{r}\right)}
$$

or

$$
\frac{\left\{\bar{q}_{0}\right\}_{R_{j}}}{\left\{\bar{w}_{0}\right\}_{R_{j}}} \rightarrow-\frac{K}{L}=i \frac{K}{L^{*}} \text { as } \quad k_{R_{j}} r \rightarrow \infty
$$

Thus at horizontal ranges large compared to the wavelength, the surface displacements are either prograde elliptical or retrograde elliptical dependent on whether the real ratio $\left(K / L^{*}\right)$ is positive or negative respectively. This large distance result is the same as obtained by Haskell (1953) for the homogeneous case of plane twodimensional Rayleigh waves:

$$
\left[\frac{\dot{u}_{0}}{\dot{w}_{0}}\right]_{H}=-\frac{K}{L}
$$

Rewriting equation (79) in Haskell's notation

$$
\left\{\bar{q}_{0}\right\}_{R_{j}}=-i\left[\frac{\dot{u}_{0}}{\dot{w}_{0}}\right]_{H_{j}}\left\{\bar{w}_{0}\right\}_{R_{j}} H_{1}{ }^{(2)}\left(k_{R_{j}} r^{r}\right) / H_{0}{ }^{(2)}\left(k_{R_{j}} r\right)
$$

Evaluating the residue contributions of the integral representations for $\bar{q}_{s 1}$ $(r, \theta, D), \bar{P}_{z z_{s 1}}(r, \theta, D), \bar{P}_{r z_{s 1}}(r, \theta, D), \bar{q}_{s 2}(r, \theta, D), \quad \bar{w}_{s 2}(r, \theta, D), \bar{P}_{z z_{s 2}}(r, \theta, D) \quad$ and $\widetilde{P}_{r_{s 2}}(r, \theta, D)$ by using equations (53) and $(50)$ we find that 


$$
\begin{aligned}
& \left\{\bar{q}_{s 1}(r, \theta, D)\right\}_{R_{j}}=-i\left\{\left(A_{R_{s 1}}\right)_{12}+\left[\frac{\dot{u}_{0}}{\dot{w}_{0}}\right]_{H_{j}}\left(A_{R_{s 1}}\right)_{11}\right\} \\
& \cdot\left\{\bar{w}_{0}\right\}_{R_{1}} H_{1}{ }^{(2)}\left(k_{R_{j}} r\right) / H_{0}{ }^{(2)}\left(k_{R_{j}} r\right) \\
& \equiv-i\left[\frac{\dot{u}_{B 1}(D)}{\dot{w}_{0}}\right]_{H_{i}}\left\{\bar{w}_{0}\right\}_{R_{j}} H_{1}{ }^{(2)}\left(k_{R_{j}} r\right) / H_{0}{ }^{(2)}\left(k_{R_{j}} r\right) \\
& \left\{\bar{w}_{s 1}(r, \theta, D)\right\}_{R_{j}}=\left\{\left(A_{R_{s 1}}\right)_{22}+\left[\frac{\dot{u}_{0}}{\dot{w}_{0}}\right]_{H_{j}}\left(A_{R_{s 1}}\right)_{21}\right\}\left\{\bar{w}_{0}\right\}_{R_{j}} \\
& \equiv\left[\frac{\dot{w}_{s 1}(D)}{\dot{w}_{0}}\right]_{H_{i}}\left\{\bar{w}_{0}\right\}_{R_{i}} \\
& \left\{\bar{P}_{z z_{s 1}}(r, \theta, D)\right\}_{R_{j}}=i k_{R_{j}}\left\{\left(A_{R_{s 1}}\right)_{32}+\left[\frac{\dot{u}_{0}}{\dot{w}_{0}}\right]_{H_{j}}\left(A_{R_{s 1}}\right)_{i 1}\right\}\left\{\bar{w}_{0}\right\}_{R_{j}} \\
& \equiv i k_{R_{j}}\left[\frac{\sigma_{\mathrm{s} 1}(D)}{\frac{\dot{w}_{0}}{c}}\right]_{H_{j}}\left\{\bar{w}_{0}\right\}_{R_{j}} \\
& \left\{\bar{P}_{r z_{s 1}}(r, \theta, D)\right\}_{R_{j}}=k_{R_{j}}\left\{\left(A_{R_{s 1}}\right)_{42}+\left[\frac{\dot{u}_{0}}{\dot{u}_{0}}\right]_{H_{j}}\left(A_{R_{s 1}}\right)_{41}\right\} \\
& \times\left\{\bar{w}_{0}\right\}_{R_{j}} H_{1}{ }^{(2)}\left(k_{R_{j}} r\right) / H_{0}{ }^{(2)}\left(k_{R_{j}}{ }^{r}\right) \\
& \equiv k_{R_{j}}\left[\frac{\tau_{s 1}(D)}{\frac{\dot{w}_{0}}{c}}\right]_{H_{j}}\left\{\bar{w}_{0}\right\}_{R_{j}} H_{1}{ }^{(2)}\left(k_{R_{j}} r\right) / H_{0}{ }^{(2)}\left(k_{R_{j}} r\right)
\end{aligned}
$$

and

$$
\begin{gathered}
\left\{\bar{q}_{s 2}(r, \theta, D)\right\}_{R_{j}}=\left\{\bar{q}_{s 1}(r, \theta, D)\right\}_{R_{j}} \\
\left\{\bar{w}_{s 2}(r, \theta, D)\right\}_{R_{j}}=\left\{\bar{w}_{s 1}(r, \theta, D)\right\}_{R_{j}} \\
\left\{\bar{P}_{z_{s} s}(r, \theta, D)\right\}_{R_{j}}=\left\{\bar{P}_{z z_{s 1}}(r, \theta, D)\right\}_{R_{j}} \\
\left\{\bar{P}_{r_{z_{s}}}(r, \theta, D)\right\}_{R_{j}}=\left\{\bar{P}_{r z_{s 1}}(r, \theta, D)\right\}_{R_{j}}
\end{gathered}
$$

From the set of equations (81), we see that there is no discontinuity in displacement or stress across the source plane $z=D$, for the residue contributions. Thus from equation (80) we obtain 


$$
\begin{aligned}
\left\{\bar{q}_{m}(r, \theta, z)\right\} & =-i\left[\frac{\dot{u}_{m}(z)}{\dot{w}_{0}}\right]_{H_{j}}\left\{\bar{w}_{0}\right\}_{R_{i}} H_{1}{ }^{(2)}\left(k_{R_{i}} r\right) / H_{0}{ }^{(2)}\left(k_{R_{j}}{ }^{r}\right) \\
\left\{\bar{w}_{m}(r, \theta, z)\right\} & =\left[\frac{\dot{w}_{m}(z)}{\dot{w}_{0}}\right]_{H_{j}}\left\{\bar{w}_{0}\right\}_{R_{i}} \\
\left\{\bar{P}_{z z_{m}}(r, \theta, z)\right\} & =i k_{R_{j}}\left[\frac{\sigma_{m}(z)}{\frac{\dot{w}_{0}}{c}}\right]_{H_{j}}\left\{\bar{w}_{0}\right\}_{R_{j}} \\
\left\{\bar{P}_{r z_{m}}(r, \theta, z)\right\} & =k_{R_{j}}\left[\frac{\tau_{m}(z)}{\frac{\dot{w}_{0}}{c}}\right]_{H_{j}}\left\{\bar{w}_{0}\right\}_{R_{j}} H_{1}{ }^{(2)}\left(k_{R_{j}} r\right) / H_{0}{ }^{(2)}\left(k_{R_{j}} r\right)
\end{aligned}
$$

where the homogeneous ratios ( $H$ subscripts) are given for all $m$ in terms of

$$
A_{R_{m}}(z)=\mathfrak{Q}_{R_{m}}(z) \mathfrak{Q}_{R_{m-1}} \cdots \mathfrak{Q}_{R_{1}}
$$

and where $\mathfrak{Q}_{R_{m}}(z)$ is the layer matrix for a sublayer in $m$ of thickness $d_{m}(z)=$ $z-z_{m-1}$.

From equation (58) and the definitions of the homogeneous ratios implied in equations $(80)$ and $(82)$ we have

$$
\left[Y+\frac{K}{L} Z\right]=-\left\{\delta\left(\frac{\dot{w}_{R_{s}}}{c}\right)\left[\frac{\sigma_{s}(D)}{\frac{\dot{w}_{0}}{c}}\right]_{H_{j}}+\delta \tau_{R_{s}}\left[\frac{\dot{u}_{s}(D)}{\dot{w}_{0}}\right]_{H_{j}}\right\}
$$

Inserting real quantities (asterisked) and using equations (44), (50) and (75) yields

$$
\frac{N_{R_{j}}^{(I)}}{\left(\frac{\partial F_{R}}{\partial k}\right)_{\omega, j}}=i k_{R_{j}} a_{s}^{3} \bar{p}_{0_{s}} \frac{\left\{\frac{1}{2 \mu_{s}}\left[\frac{\sigma_{s}^{*}(D)}{\frac{\dot{w}_{0}}{c}}\right]_{H_{j}}-\left[\frac{\dot{u}_{s}(D)}{\dot{w}_{0}}\right]_{H_{j}}\right\}}{\left[\left(1-\frac{a_{s}^{2} k_{\beta_{s}}^{2}}{4}\right)^{2}+k_{\alpha_{s}}^{2}{a_{s}}^{2}\right]^{1 / 2}} \mathbf{A}_{R_{j}}(\omega) e^{i\left(k_{\alpha_{s}} a_{s}-\theta_{S P}\right)}
$$

where

$$
\mathbf{A}_{R_{j}}(\omega)=\frac{\left[G^{*} N-L^{*} H\right]}{\left(\frac{\partial \bar{F}_{R}}{\partial k}\right)_{\omega, j}}
$$

Therefore the Fourier time transformed Rayleigh wave surface displacements for an explosive spherical source at depth $D$ are 


$$
\begin{aligned}
& \left\{\bar{w}_{0}\right\}_{R_{j}}=i k_{R_{j}} \bar{p}_{0 s} a_{s}{ }^{3} \frac{\left\{\frac{1}{2 \mu_{s}}\left[\frac{\sigma_{s}{ }^{*}(D)}{\frac{\dot{w}_{0}}{c}}\right]_{H_{j}}-\left[\frac{\dot{u}_{s}^{*}(D)}{\dot{w}_{0}}\right]_{H_{j}}\right\}}{\left[\left(1-\frac{a_{s}{ }^{2} k_{\beta_{s}}^{2}}{4}\right)^{2}+k_{\alpha_{s}}^{2} a_{s}{ }^{2}\right]^{1 / 2}} \mathbf{A}_{R_{j}} e^{i\left(k_{\alpha_{s} a_{s}}{ }^{-\theta_{S} P}\right)} \\
& \times H_{0}{ }^{(2)}\left(k_{R_{j}} r\right) \\
& \left\{\bar{q}_{0}\right\}_{R_{j}}=\left[\frac{\dot{u}_{0}^{*}}{\dot{w}_{0}}\right]_{H_{j}} i \pi k_{R_{i}} \bar{p}_{0 s} a_{s}{ }^{3} \frac{\left\{\frac{1}{2 \mu_{s}}\left[\frac{\sigma_{s}^{*}(D)}{\frac{\dot{u}_{0}}{c}}\right]_{H_{j}}-\left[\frac{u_{s}^{*}(D)}{\dot{w}_{0}}\right]_{H_{j}}\right\}}{\left[\left(1-\frac{a_{s}^{2} k_{\beta_{s}}^{2}}{4}\right)^{2}+k_{\alpha_{s}}^{2} a_{s}^{2}\right]^{1 / 2}} \\
& \times \mathbf{A}_{R_{j}} e^{i\left(k_{\alpha_{s}} a_{s}-\theta_{S P}\right)} H_{1}{ }^{(2)}\left(k_{R_{j}}{ }^{p}\right)
\end{aligned}
$$

Before proceeding to the next section, it should be noted that by means of equations (46), the part of our solution dependent on source depth $D$ can be written as

$$
\left\{\frac{1}{2 \mu_{s}}\left[\frac{\sigma_{s}^{*}(D)}{\frac{\dot{w}_{0}}{c}}\right]_{H_{j}}-\left[\frac{u_{s}^{*}(D)}{\dot{w}_{0}}\right]_{H_{j}}\right\}=-\frac{k^{2}}{\gamma_{s}}\left[\frac{\varphi_{s}(D)}{\frac{\dot{w}_{0}}{c}}\right]_{H_{j}}
$$

Rayleigh Waves from a Vertical Point Force at Depth

Consider the same elastic medium as before but with a vertical point force in layer $s$ at $(0, \theta, D)$. The Fourier time transformed vertical point force $\bar{L}(\omega)$, positive in the downward or positive $z$ direction is defined (Pekeris, 1955) in terms of the transformed normal stress to $z=D$ plane as

$$
2 \pi \int_{0}^{\infty}\left[\bar{P}_{z z_{s}}\left(r, \theta, D^{+}\right)-\bar{P}_{z z_{s}}\left(r, \theta, D^{-}\right)\right] r d r=-\bar{L}
$$

or

$$
\left[\bar{P}_{z z_{s}}\left(r, \theta, D^{+}\right)-\bar{P}_{z \hat{s}_{s}}\left(r, \theta, D^{-}\right)=-\frac{\bar{L}}{2 \pi} \int_{0}^{\infty} J_{0}(k r) k d k\right.
$$

with continuous $\bar{q}_{s}, \bar{w}_{s}$ and $\bar{P}_{r_{s}}$ for all $r$ along $z=D$.

Since this source is azimuthally symmetric about the $z$ axis and the boundary conditions are at the $z$ constant plane interfaces this problem reduces, as before, to cylindrical symmetry. Comparing equation (88) with equation (46), we obtain

$$
\begin{aligned}
\delta\left(\frac{\dot{u}_{R_{s}}}{c}\right) & =0 \\
\delta\left(\frac{\dot{w}_{R_{s}}}{c}\right) & =0 \\
\delta \sigma_{R_{s}} & =-\frac{\bar{L} k}{2 \pi}
\end{aligned}
$$




$$
\begin{aligned}
\delta \tau_{R_{\varepsilon}} & =0 \\
\delta\left(\frac{\dot{v}_{L_{s}}}{c}\right) & =0 \\
\delta \tau_{L_{s}} & =0
\end{aligned}
$$

Thus the vector equation in the source layer $s$ for the vertical point force is

$$
\left[\begin{array}{c}
\frac{\dot{u}_{R_{s 2}}(D)}{c} \\
\frac{\dot{w}_{R_{s 2}}(D)}{c} \\
\sigma_{R_{s 2}}(D) \\
\tau_{R_{s 2}}(D)
\end{array}\right]=\left[\begin{array}{c}
\frac{\dot{u}_{R_{s 1}}(D)}{c} \\
\frac{\dot{w}_{R_{s 1}}(D)}{c} \\
\sigma_{R_{s 1}}(D) \\
\tau_{R_{s 1}}(D)
\end{array}\right]=\left[\begin{array}{c}
0 \\
0 \\
\delta \sigma_{R_{s}} \\
0
\end{array}\right]
$$

As an alternate method relations (89) could have been obtained from the source potentials which correspond to source definition (88) in an infinite elastic medium. The displacements due to such a force in an infinite space are

$$
\begin{aligned}
& \bar{q}_{s 0}(r, \theta, z)=\frac{\bar{L}}{4 \pi \rho_{s} \omega^{2}} \frac{\partial^{2}}{\partial r \partial z}\left(\frac{e^{-i k_{\beta_{s}} R}-e^{-i k_{\alpha_{s}} R}}{R}\right) \\
& \bar{w}_{s 0}(r, \theta, z)=\frac{\bar{L}}{4 \pi \rho_{s} \omega^{2}}\left[\frac{\partial^{2}}{\partial z^{2}}\left(\frac{e^{-i k_{\beta_{s}} R}-e^{-\imath k_{\alpha_{s}} R}}{R}\right)+k_{\beta_{s}}^{2} e^{-i k_{\beta_{s}} R}\right]
\end{aligned}
$$

Expressing equations (91) in integral form using the Sommerfeld integral and comparing with equations (46) and (30), we see that the source potentials are given by

$$
\begin{aligned}
& S_{01}^{+}=\frac{\bar{L} k}{4 \pi \rho_{s} \omega^{2}} \\
& S_{01}^{-}=-\frac{\bar{L} k}{4 \pi \rho_{s} \omega^{2}} \\
& S_{02}^{+}=S_{02}^{-}=-i \frac{\bar{L}}{4 \pi \rho_{s} \omega^{2} r_{\beta}} \\
& S_{03}^{+}=S_{03}^{-}=0
\end{aligned}
$$

Substituting equations (92) into relations (37) and (40), we obtain equations (89) again. In the next section where we consider the horizontal point force we will make use of equations (91) to obtain our source definition. 
Following the same procedure as for the explosive source, we obtain

$$
\begin{aligned}
\frac{\dot{u}_{R_{0}}}{c} & =W-\delta \sigma_{R_{s}}\left(A_{R_{s 1}}^{-1}\right)_{13} \\
\frac{\dot{w}_{R_{0}}}{c} & =X-\delta \sigma_{R_{s}}\left(A_{R_{s 1}}^{-1}\right)_{23} \\
Y & =\delta \sigma_{R_{s}}\left(A_{R_{s 1}}^{-1}\right)_{33} \\
Z & =\delta \sigma_{R_{s}}\left(A_{R_{s 1}}^{-1}\right)_{43}
\end{aligned}
$$

and by equation (57), the inverse of $A_{R_{s 1}}$,

$$
\begin{aligned}
\frac{\dot{u}_{R_{0}}}{c} & =W-\delta \sigma_{R_{s}}\left(A_{R_{s 1}}\right)_{24} \\
\frac{\dot{w}_{R_{0}}}{c} & =X+\delta \sigma_{R_{s}}\left(A_{R_{s 1}}\right)_{23} \\
Y & =\delta \sigma_{R_{s}}\left(A_{R_{s 1}}\right)_{22} \\
Z & =-\delta \sigma_{R_{s}}\left(A_{R_{s 1}}\right)_{21}
\end{aligned}
$$

Using equations (59) through (71) yields

$$
\frac{\dot{w}_{R_{0}}}{c}=-\frac{N_{R}^{(1)} N_{R}{ }^{(2)}}{F_{R}}
$$

and

$$
\frac{\dot{u}_{R_{0}}}{c}=\frac{N_{R}{ }^{(3)} N_{R}{ }^{(4)}}{F_{R}}
$$

where from equations (94)

$$
\begin{aligned}
& N_{R}{ }^{(2)}=1-\frac{F_{R}}{N_{R}{ }^{(1)}} \delta \sigma_{R_{\varepsilon}}\left(A_{R_{s 1}}\right)_{23} \\
& N_{R}{ }^{(4)}=1-\frac{F_{R}}{N_{R}{ }^{(3)}} \delta \sigma_{R_{s}}\left(A_{R_{s 1}}\right)_{24}
\end{aligned}
$$

As before the displacements at the free surface are

$$
\vec{w}_{0}=i \int_{0}^{\infty} \frac{1}{k} \frac{N_{R}^{(1)} N_{R}{ }^{(2)}}{F_{R}} J_{0}(k r) d k
$$


and

$$
\bar{q}_{0}=-\int_{0}^{\infty} \frac{1}{k} \frac{N_{R}^{(3)} N_{R}^{(4)}}{F_{R}} J_{1}(k r) d k
$$

The residue contribution of equations (98) and (99) are thus

$$
\begin{aligned}
\left\{\bar{w}_{0}\right\}_{R_{j}} & =\frac{\pi}{k_{R_{j}}} \frac{N_{R_{j}}^{(1)}}{\left(\frac{\partial F_{R}}{\partial k}\right)_{\omega, j}} H_{0}^{(2)}\left(k_{R_{j}} r\right) \\
\left\{\bar{q}_{0}\right\}_{R_{j}} & =i \frac{K}{L} \frac{\pi}{k_{R_{j}}} \frac{N_{R_{i}}^{(1)}}{\left(\frac{\partial \bar{F}_{R}}{\partial k}\right)_{\omega, j}} H_{1}{ }^{(2)}\left(k_{R_{j}} r\right)
\end{aligned}
$$

where $N_{R_{i}}^{(1)}$ is, as before,

$$
N_{R_{j}}^{(1)}=[G N-L H]\left\{Y+\frac{K}{L} Z\right\}
$$

but now from equation (94)

$$
N_{R_{i}}^{(1)}=[G N-L H] \delta \sigma_{R_{\mathrm{s}}}\left\{\left(A_{R_{81}}\right)_{22}-\frac{K}{L}\left(A_{R_{s 1}}\right)_{21}\right\}
$$

All the relations concerning $F_{R}$ and the displacements and stresses at depth in terms of the homogeneous solutions shown for the explosive source are also true for the vertical point force. With these relations $N_{R_{j}}^{(1)}$ can be rewritten as

$$
N_{R_{j}}^{(1)}=-\frac{\bar{L} k_{R_{j}}}{2 \pi}[G N-L H]\left[\frac{\dot{w}_{s}(D)}{\dot{w}_{0}}\right]_{H_{j}}
$$

Therefore the Fourier time transformed Rayleigh wave displacements for a vertical point force at depth $D$ are

$$
\begin{aligned}
\left\{\bar{w}_{0}\right\}_{R_{j}} & =-i \frac{\bar{L}}{2}\left[\frac{\dot{w}_{s}(D)}{\dot{w}_{0}}\right]_{H_{j}} \mathbf{A}_{R_{j}} H_{0}{ }^{(2)}\left(k_{R_{j}} r\right) \\
\left\{\bar{q}_{0}\right\}_{R_{j}} & =-i \frac{\bar{L}}{2}\left[\frac{\dot{u}_{0}{ }^{*}}{\dot{w}_{0}}\right]_{H_{j}}\left[\frac{\dot{w}_{s}}{\dot{w}_{0}}\right]_{H_{j}} \mathbf{A}_{R_{j}} H_{1}{ }^{(2)}\left(k_{R_{j}} r\right)
\end{aligned}
$$

where, as in equation (84),

$$
\mathbf{A}_{R_{i}}=\frac{\left[G^{*} N-L^{*} H\right]}{\left(\frac{\partial F_{R}}{\partial t}\right)_{\omega, j}}
$$




\section{Rayleigh and Love Waves from a Horizontal Point Force at Depth}

We now consider a Fourier time transformed horizontal point force in layer $s$ at depth $D$ directed in the $\theta=0$ direction. As our source displacement field, we use the displacements due to a horizontal point force of strength $\bar{L}(\omega)$ in an elastic space with the same elastic properties as layer $s$. These displacements are obtained by expressing the source displacement field (91) of the previous section in terms of a cylindrical axis at right angles to the force (figure 2 ).

From equations (91) the source displacement field in the new coordinates is given by

$$
\begin{aligned}
\bar{q}_{s 0}(r, \theta, z) & =\frac{\bar{L}}{4 \pi \omega^{2} \rho_{s}} \cos \theta\left[\frac{\partial}{\partial r^{2}}\left(\frac{e^{-i k_{\beta_{s}} R}-e^{i k_{\alpha_{s}} R}}{R}\right)+k_{\beta_{s}}^{2} \frac{e^{-i k_{\beta_{s}} R}}{R}\right] \\
\bar{v}_{s 0}(r, \theta, z) & =\frac{\bar{L}}{4 \pi \omega^{2} \rho_{s}} \sin \theta\left[\frac{1}{r} \frac{\partial}{\partial r}\left(\frac{e^{-i k_{\beta_{s}} R}-e^{-i k_{\alpha_{s}} R}}{R}\right)+k_{\beta_{s}}^{2} e^{-i k_{\beta_{s}} R}\right] \\
\bar{w}_{s 0}(r, \theta, z) & =\frac{\bar{L}}{4 \pi \omega^{2} \rho_{s}} \cos \theta \frac{\partial^{2}}{\partial z \partial r}\left(\frac{e^{-i k_{\beta_{s}} R}-e^{-i k_{\alpha_{s} R} R}}{R}\right)
\end{aligned}
$$

where

$$
R^{2}=r^{2}+(z-D)^{2}
$$

Rewriting as an integral representation, yields

$$
\begin{aligned}
& \bar{q}_{s 0}(r, \theta, z)=-i \frac{\bar{L}}{4 \pi \omega^{2} \rho_{s}} \cos \theta \int_{0}^{\infty}[ k^{2}\left(\frac{e^{-i k r_{\alpha_{s}}|z-D|}}{r_{\alpha_{s}}}+r_{\beta_{s}} e^{-i k r_{\beta_{s}}|z-D|}\right) \\
&\left.\cdot \frac{d J_{1}(k r)}{d k r}+k_{\beta_{s}}^{2} e^{i k r_{\beta_{s}}|z-D|} \frac{J_{1}(k r)}{k r}\right] d k \\
& \bar{v}_{s 0}(r, \theta, z)=i \frac{\bar{L}}{4 \pi \omega^{2} \rho_{s}} \sin \theta \int_{0}^{\infty}\left[k^{2}\left(\frac{e^{-i k r_{\alpha_{s}}|z-D|}}{r^{2} \alpha_{s}}+r_{\beta_{s}} e^{-i k r_{\beta_{s}}|z-D|}\right)\right. \\
&\left.\cdot \frac{J_{1}(k r)}{k r}+k_{\beta_{s}}^{2} e^{-i k r_{\alpha_{s}}|z-D|} \frac{d J_{1}(k r)}{d k r}\right] d k
\end{aligned}
$$

$$
\begin{aligned}
\bar{w}_{s 0}(r, \theta, z)=\frac{\bar{L}}{4 \pi \omega^{2} \rho_{s}} \cos \theta\left\{\frac{|z-D|}{z-D}\right\} & \\
& \cdot \int_{0}^{\infty}\left[k^{2}\left(e^{-i k r_{\beta_{s}}|z-D|}-e^{-i k r_{\alpha_{s}}|z-D|}\right) J_{1}(k r)\right] d k
\end{aligned}
$$

Comparing the source $r$ and $\theta$ dependence with equations ( 7 ), we see that the $r$ and $\theta$ dependence of the solution integrands are given by equations (7) when 
$l=1$, and that

$$
\begin{aligned}
& \frac{\dot{u}_{R_{s} 0}(z)}{c}=-i \frac{\dot{L}}{4 \pi \omega^{2} \rho_{s}} k^{3}\left(\frac{e^{-i k r_{\alpha_{s}}|z-D|}}{r_{\alpha_{s}}}+r_{\beta_{s}} e^{-i k r_{\beta_{s}}|z-D|}\right) \\
& \frac{\dot{v}_{L_{s 0}}(z)}{c}=\frac{\bar{L}}{4 \pi \omega^{2} \rho_{s}} k k_{\beta_{s}}^{2} e^{-i k r_{\beta_{s}}|z-D|} \\
& \frac{\dot{u}_{R_{s 0}}(z)}{c}=i \frac{\bar{L}}{4 \pi \omega^{2} \rho_{s}}\left\{\frac{|z-D|}{z-\bar{D}}\right\} k^{3}\left(e^{-i k r_{\beta_{s}}|z-D|}-e^{-i k r_{\alpha_{s}} ; z-D \mid}\right)
\end{aligned}
$$

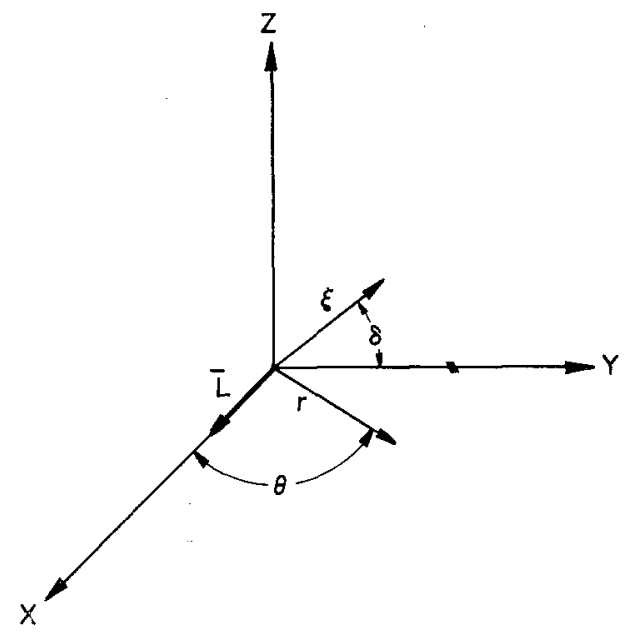

Fig. 2. Horizontal force geometry.

Evaluating the source stresses from equations (105) and comparing the integrands with equations ( 7 ), we obtain

$$
\begin{aligned}
& \sigma_{R_{s 0}}(z)=-i \frac{k \bar{L}}{4 \pi}\left[\left(\gamma_{s}-1\right) \frac{e^{-i k r_{\alpha_{s}}|z-D|}}{r_{\alpha_{s}}}-\gamma_{s} \gamma_{\beta_{s}} e^{-i k r_{\beta_{s}}|z-D|}\right] \\
& \tau_{R_{s 0}}(z)=i \frac{k \bar{L}}{4 \pi}\left\{\frac{|z-D|}{z-D}\right\}\left[\gamma_{s} e^{-i k r_{\alpha_{s}}|z-D|}-\left(\gamma_{s}-1\right) e^{-i k r_{\beta_{s}}|z-D|}\right] \\
& \tau_{L_{s 0}}(z)=-\frac{k \bar{L}}{4 \pi}\left\{\frac{|z-D|}{z-D}\right\} e^{-i k r \beta_{s}|z-D|}
\end{aligned}
$$

Evaluation of equations (106) and (107) leads to the vector equations for the source layer at $z=D$, 


$$
\left[\begin{array}{c}
\frac{\dot{u}_{R_{s 2}}(D)}{c} \\
\frac{\dot{w}_{R_{s 2}}(D)}{c} \\
\sigma_{R_{s 2}}(D) \\
\tau_{R_{s 2}}(D)
\end{array}\right]=\left[\begin{array}{c}
\frac{\dot{u}_{R_{s 1}}(D)}{c} \\
\frac{\dot{w}_{R_{s 1}}(D)}{c} \\
\sigma_{R_{s 1}}(D) \\
\tau_{R_{s 1}}(D)
\end{array}\right]+\left[\begin{array}{c}
0 \\
0 \\
0 \\
\delta \tau_{R_{s}}
\end{array}\right]
$$

where

$$
\delta \tau_{R_{s}}=i \frac{k \bar{L}}{2 \pi}
$$

and

$$
\left[\begin{array}{c}
\frac{\dot{v}_{L_{s 2}}(D)}{c} \\
\tau_{L_{s 2}}(D)
\end{array}\right]=\left[\begin{array}{c}
\frac{\dot{v}_{L_{s 1}}(D)}{c} \\
\tau_{L_{s 1}}(D)
\end{array}\right]+\left[\begin{array}{c}
0 \\
\delta \tau_{L_{s}}
\end{array}\right]
$$

where

$$
\delta \tau_{L_{s}}=-\frac{k \bar{L}}{2 \pi}
$$

The same result can be derived by noting that the source displacements can be obtained from the source potentials

$$
\begin{aligned}
& \varphi_{s 0}(z)=-i \frac{\mid \bar{L}}{4 \pi \omega^{2} \rho_{s}} \frac{e^{-i k r_{\alpha_{s}}|z-D|}}{r_{\alpha_{s}}} \\
& \psi_{s 0}(z)=\frac{\bar{L}}{4 \pi \omega^{2} \rho_{s}}\left\{\frac{|z-D|}{z-D}\right\} e^{-i k r_{\beta_{s}}|z-D|} \\
& \chi_{s 0}(z)=-i \frac{\bar{L}}{4 \pi \omega^{2} \rho_{s}} \frac{k_{\beta_{s}}^{2}}{k r_{\beta_{s}}} e^{-i k r_{\beta_{s}}|z-D|}
\end{aligned}
$$

And by comparison with equations (30) we have

$$
\begin{aligned}
& S_{01}^{+}=S_{01}^{-}=-i \frac{\bar{L}}{4 \pi \omega^{2} \rho_{s}} \frac{k}{r_{\alpha_{s}}} \\
& S_{02}^{+}=-S_{02}^{-}=\frac{\bar{L}}{4 \pi \omega^{2} \rho_{s}} \\
& S_{03}^{+}=S_{03}=-i \frac{\bar{L}}{4 \pi \omega^{2} \rho_{s}} \frac{k \beta_{s}}{k r_{\beta_{s}}}
\end{aligned}
$$

Substitution of equations (111) into equations (37) and (40) yields the same result as above. 


$$
\delta \tau_{R_{s}}=i \frac{k \bar{L}}{2 \pi} \text { and } \delta \tau_{L_{s}}=-\frac{k \bar{L}}{2 \pi}
$$

The horizontal force problem has now separated into two sets of independent vector relations. The first set, given by equations (9a), (15) and (108), represents Rayleigh type surface waves; the second set, (9b), (24) and (109), Love type surface waves when the residue contributions are obtained. Following the procedure of the previous sections, we obtain for the first set

$$
\begin{aligned}
\frac{\dot{u}_{R_{0}}}{c} & =W+\delta \tau_{R_{s}}\left(A_{R_{s 1}}\right)_{14} \\
\frac{\dot{w}_{R_{0}}}{c} & =X-\delta \tau_{R_{s}}\left(A_{R_{s 1}}\right)_{13} \\
Y & =-\delta \tau_{R_{s}}\left(A_{R_{s 1}}\right)_{12} \\
Z & =\delta \tau_{R_{s}}\left(A_{R_{s 1}}\right)_{11}
\end{aligned}
$$

Solving equations (112) yields

$$
\frac{\dot{w}_{R_{0}}}{c}=-\frac{N_{R}^{(1)} N_{R}^{(2)}}{F_{R}}
$$

and

$$
\frac{\dot{u}_{R_{0}}}{c}=\frac{N_{R}{ }^{(3)} N_{R}{ }^{(4)}}{F_{R}}
$$

where

$$
\begin{aligned}
& N_{R}{ }^{(2)}=1+\frac{F_{R}}{N_{R}{ }^{(1)}} \delta \tau_{R_{s}}\left(A_{R_{s 1}}\right)_{13} \\
& N_{R}{ }^{(4)}=1+\frac{F_{R}}{N_{R}{ }^{(3)}} \delta \tau_{R_{s}}\left(A_{R_{s 1}}\right)_{14}
\end{aligned}
$$

For the second set, we have similar to equations (51) and (52)

$$
\left[\begin{array}{c}
\frac{\dot{v}_{L_{n-1}}\left(z_{n-1}\right)}{c} \\
\tau_{L_{n-1}}\left(z_{n-1}\right)
\end{array}\right]=A_{L}{ }^{s 2}\left[\begin{array}{l}
\frac{\dot{v}_{L_{s 2}}(D)}{c} \\
\tau_{L_{s 2}}(D)
\end{array}\right]
$$

and

$$
\left[\begin{array}{c}
\frac{\dot{v}_{L_{s 1}}(D)}{c} \\
\tau_{L_{s 1}}(D)
\end{array}\right]=A_{L_{s 1}}\left[\begin{array}{c}
\frac{\dot{v}_{L_{1}}(D)}{c} \\
0
\end{array}\right]
$$


where

$$
\begin{aligned}
A_{L}{ }^{s 2} & =\mathfrak{Q}_{L_{n-1}} \cdots Q_{L_{s 2}} \\
A_{L_{s 1}} & =\mathfrak{Q}_{L_{s 1}} \cdots \mathfrak{Q}_{L_{1}}
\end{aligned}
$$

and

$$
\frac{\dot{v}_{L_{0}}}{c}=\frac{\dot{v}_{L_{1}}(0)}{c}
$$

Using the following definition

$$
\left[\begin{array}{c}
V \\
T
\end{array}\right]=A_{L_{s 1}}^{-1}\left[\begin{array}{c}
\frac{\dot{v}_{L_{s 2}}(D)}{c} \\
\tau_{L_{s 2}}(D)
\end{array}\right]
$$

and multiplying the source layer vector equation (109) by $A_{L_{s 1}}^{-1}$ yields

$$
\left[\begin{array}{c}
V \\
T
\end{array}\right]=\left[\begin{array}{c}
\frac{\dot{v}_{L_{0}}}{c} \\
0
\end{array}\right]+A_{L_{s 1}}^{-1}\left[\begin{array}{c}
0 \\
\delta \tau_{L_{s}}
\end{array}\right]
$$

or

$$
\begin{aligned}
\frac{\dot{v}_{L_{0}}}{c} & =V-\delta \tau_{L_{s}}\left(A_{L_{s 1}}^{-1}\right)_{12} \\
T & =\delta \tau_{L_{s}}\left(A_{L_{s 1}}^{-1}\right)_{22}
\end{aligned}
$$

Using relation (29), equations (119) can be rewritten as

$$
\begin{aligned}
\frac{\dot{v}_{L_{0}}}{c} & =V+\delta \tau_{L_{s}}\left(A_{L_{s 1}}\right)_{12} \\
T & =\delta \tau_{L_{s}}\left(A_{L_{s 1}}\right)_{11}
\end{aligned}
$$

From equation (26), we have for the half-space or layer $n$

$$
\left[\begin{array}{c}
\hat{\epsilon}_{n}{ }^{\prime}+\hat{\epsilon}_{n}{ }^{\prime \prime} \\
\hat{\epsilon}_{n}{ }^{\prime}-\hat{\epsilon}_{n}{ }^{\prime \prime}
\end{array}\right]=E_{L_{n}}^{-1}\left[\begin{array}{c}
\frac{\dot{v}_{L_{n}}\left(z_{n-1}\right)}{c} \\
\tau_{L_{n}}\left(z_{n-1}\right)
\end{array}\right]
$$

Our boundary conditions at infinity in the half-space as before requires that $\hat{\epsilon}_{n}^{\prime \prime}$ $=0$. Defining 


$$
A_{L}=A_{L}^{s 2} A_{L_{s 1}}=Q_{L_{n-1}} \cdots Q_{L_{s 2}} Q_{L_{s 1}} \cdots Q_{L_{1}}=Q_{L_{n-1}} \cdots Q_{L_{s}} \cdots Q_{L_{1}}
$$

where it can be shown that $\mathscr{Q}_{L_{s 2}} \mathfrak{Q}_{L_{s 1}}=\mathscr{Q}_{L_{s}}$, we obtain using equations (121), (116) and (117)

$$
\left[\begin{array}{c}
\hat{\boldsymbol{\epsilon}}_{n}{ }^{\prime} \\
\hat{\epsilon}_{n}{ }^{\prime}
\end{array}\right]=E_{L_{n}}^{-1} A_{L}\left[\begin{array}{c}
V \\
T
\end{array}\right]
$$

or

$$
V=-\frac{\left[\left(J_{L}\right)_{12}-\left(J_{L}\right)_{22}\right]}{\left[\left(J_{L}\right)_{11}-\left(J_{L}\right)_{21}\right]} T
$$

where

$$
J \equiv E_{L_{n}}^{-1} A_{L}=\left[\begin{array}{cc}
(i k)^{-1} & 0 \\
0 & -\left(i k \mu_{n} r_{\beta_{n}}\right)^{-1}
\end{array}\right]\left[\begin{array}{c}
\left(A_{L}\right)_{11}\left(A_{L}\right)_{12} \\
\left(A_{L}\right)_{21}\left(A_{L}\right)_{22}
\end{array}\right]
$$

Evaluation of equation (124) and substitution of equation (123) in (120) yields

$$
\frac{\dot{v}_{L_{0}}}{c}=\frac{N_{L}^{(1)} N_{L}^{(2)}}{F_{L}}
$$

where

$$
\begin{aligned}
F_{L} & =-\left(A_{L}\right)_{21}^{*}-\left(A_{L}\right)_{11} \mu_{n} r_{\beta_{n}}^{*} \\
N_{L}{ }^{(1)} & =i \frac{k \bar{L}}{2 \pi}\left[\left(A_{L}\right)_{22}-\left(A_{L}\right)_{12}^{*} \mu_{n} r_{\beta_{n}}^{*}\right]\left(A_{L_{s 1}}\right)_{11}
\end{aligned}
$$

and

$$
N_{L}^{(2)}=1-i \frac{k \bar{L}}{2 \pi} \frac{F_{L}}{N_{L}^{(1)}}\left(A_{L_{s 1}}\right)_{21}^{*}
$$

since

$$
\delta \tau_{L_{s}}=-\frac{k \bar{L}}{2 \pi}
$$

The matrix elements of $\mathfrak{Q}_{L_{m}}$ are real or imaginary by the same criteria as $\mathfrak{Q}_{R_{m}}$. These criteria will also hold for the matrix product elements. In addition for $c \leqq \beta_{n}$ we have as before that $r_{\beta_{n}}$ is imaginary. The imaginary quantities are expressed as real quantities (designated by asterisks) multiplied by $i$. This notation was used in equations (126). 
$F_{L}(\omega, k)=0$ is a form of the period equation for Love wave propagation in plane multilayered isotropic solids. Since we are interested in the residue contribution we note that when $F_{L}=0$, equations (126) reduce to

$$
\begin{aligned}
& \mu_{n} r_{\beta_{n}}^{*}=-\frac{\left(A_{L}\right)_{21}^{*}}{\left(A_{L}\right)_{11}} \\
& N_{L}{ }^{(2)}=1
\end{aligned}
$$

and

$$
N_{L}{ }^{(1)}=i \frac{k \bar{L}}{2 \pi} \frac{\left[\left(A_{L}\right)_{11}\left(A_{L}\right)_{22}+\left(A_{L}\right)_{12}^{*}\left(A_{L}\right)_{21}^{*}\right]}{\left(A_{L}\right)_{11}}\left(A_{L_{s 1}}\right)_{11}
$$

And again using relation (29), we can show that

$$
\left(A_{L}\right)_{11}\left(A_{L}\right)_{22}+\left(A_{L}\right)_{12}^{*}\left(A_{L}\right)_{21}^{*}=1
$$

and

$$
N_{L}^{(1)}=i \frac{k \bar{L}}{2 \pi} \frac{1}{\left(A_{L}\right)_{11}}\left(A_{L_{s 1}}\right)_{11}
$$

Thus be equations (113), (114), (128) and (7) for $m=1$, the Fourier displacements at the free surface are

$$
\begin{gathered}
\bar{q}_{0} \equiv \bar{q}_{1}(r, \theta, 0)=\int_{0}^{\infty}\left\{\frac{1}{k} \frac{N_{R}{ }^{(3)} N_{R}{ }^{(4)}}{F_{R}} \frac{d J_{1}(k r)}{d k r}-\frac{i}{k} \frac{N_{L}{ }^{(1)} N_{L}{ }^{(2)}}{F_{L}} \frac{J_{1}(k r)}{k r}\right\} d k \cos \theta \\
\bar{v}_{0} \equiv \bar{v}_{1}(r, \theta, 0)= \\
\int_{0}^{\infty}\left\{-\frac{1}{k} \frac{N_{R}{ }^{(3)} N_{R}{ }^{(4)}}{F_{R}} \frac{J_{1}(k r)}{k r}+\frac{i}{k} \frac{N_{L}{ }^{(1)} N_{L}{ }^{(2)}}{F_{L}} \frac{d J_{1}(k r)}{d k r}\right\} d k \sin \theta \\
\bar{w}_{0} \equiv \bar{w}_{1}(r, \theta, 0)=\int_{0}^{\infty} \frac{i}{k} \frac{N_{R}{ }^{(1)} N_{R}{ }^{(2)}}{F_{R}} J_{1}(k r) d k \cos \theta
\end{gathered}
$$

Evaluating the residue contribution of equations (129) for the zeros of $F_{R}$ we obtain for each $j$ th mode

$$
\begin{aligned}
\left\{\bar{w}_{0}\right\}_{R_{j}} & =\frac{\pi}{k_{R_{i}}} \frac{N_{R_{j}}^{(1)} N_{R_{j}}^{(2)}}{\left(\frac{\partial F_{R}}{\partial k}\right)_{\omega, j}} H_{1}^{(2)}\left(k_{R_{j}} r\right) \cos \theta \\
\left\{\bar{q}_{0}\right\}_{R_{j}} & =-i \frac{\pi}{k_{R_{j}}} \frac{N_{R_{j}}^{(3)} N_{R_{j}}^{(4)}}{\left(\frac{\partial F_{R}}{\partial k}\right)_{\omega, j}}\left\{H_{0}^{(2)}\left(k_{R_{j}} r^{\circ}\right)-\frac{H_{1}^{(2)}\left(k_{R_{j}} r\right)}{k_{R_{j}} r}\right\} \cos \theta \\
\left\{\bar{v}_{0}\right\}_{R_{j}} & =i \frac{\pi}{k_{R_{j}}} \frac{N_{R_{j}}^{(3)} N_{R_{j}}^{(4)}}{\left(\frac{\partial F_{R}}{\partial k}\right)_{\omega, j}} \frac{H_{1}^{(2)}\left(k_{R_{j}} r\right)}{k_{R_{j}} r} \sin \theta
\end{aligned}
$$


As shown before

$$
\begin{aligned}
& N_{R_{j}}^{(2)}=N_{R_{i}}^{(4)}=1 \\
& N_{R_{j}}^{(3)}=\frac{K}{L} N_{R_{j}}^{(1)}=-i\left[\frac{\dot{u}_{0}^{*}}{\dot{w}_{0}}\right]_{H_{i}} N_{R_{j}}^{(1)} \\
& N_{R_{j}}^{(1)}=[G N-L H]\left\{Y+\frac{K}{L} Z\right\}
\end{aligned}
$$

and by equations (112) and (108)

$$
N_{R_{i}}^{(1)}=k_{R_{i}} \frac{\bar{L}}{2 \pi}\left[G^{*} N-L^{*} H\right]\left\{\left(A_{R_{s 1}}\right)_{12}-\frac{K}{L}\left(A_{R_{s 1}}\right)_{11}\right\}
$$

Using the homogeneous solution notation, we write equation (132) as

$$
N_{R_{i}}^{(1)}=i k_{R_{j}} \frac{\bar{L}}{2 \pi}\left[G^{*} N-L^{*} H\right]\left[\frac{\dot{u}_{s}^{*}(D)}{\dot{w}_{0}}\right]_{H_{i}}
$$

yielding the Rayleigh contribution for a horizontal point force at depth $D$

$$
\begin{aligned}
\left\{\bar{w}_{0}\right\}_{R_{j}} & =i \frac{\bar{L}}{2}\left[\frac{\dot{u}_{s}{ }^{*}(D)}{\dot{w}_{0}}\right]_{H_{j}} \mathbf{A}_{R_{j}} H_{1}{ }^{(2)}\left(k_{R_{j}} r\right) \cos \theta \\
\left\{\bar{q}_{0}\right\}_{R_{j}} & =-i \frac{\bar{L}}{2}\left[\frac{\dot{u}_{0}{ }^{*}}{\dot{w}_{0}}\right]_{H_{j}}\left[\frac{\dot{u}_{s}^{*}(D)}{\dot{w}_{0}}\right]_{H_{j}} \mathbf{A}_{R_{j}}\left\{H_{0}{ }^{(2)}\left(k_{R_{j}} r\right)-\frac{H_{1}{ }^{(2)}\left(k_{R_{j}} r\right)}{k_{R_{j}} r}\right\} \cos \theta \\
\left\{\bar{v}_{0}\right\}_{R_{j}} & =i \frac{\bar{L}}{2}\left[\frac{\dot{u}_{0}{ }^{*}}{\dot{w}_{0}}\right]_{H_{j}}\left[\frac{\dot{u}_{s}^{*}(D)}{\dot{w}_{0}}\right]_{H_{j}} \mathbf{A}_{R_{j}} \frac{H_{1}{ }^{(2)}\left(k_{R_{j}} r\right)}{k_{R_{j}} r} \sin \theta
\end{aligned}
$$

where $\mathbf{A}_{R j}$ is given by equation (84).

Evaluating the residue of equations (129) for the zeros of $F_{L}$, we obtain for each $j$ th mode

$$
\begin{aligned}
& \left\{\bar{v}_{0}\right\}_{L_{j}}=\frac{\pi}{k_{L_{j}}} \frac{N_{L_{j}}^{(1)} N_{L_{j}}^{(2)}}{\left(\frac{\partial F_{L}}{\partial k}\right)_{\omega, j}}\left\{H_{0}{ }^{(2)}\left(k_{L_{j}} r\right)-\frac{H_{1}{ }^{(2)}\left(k_{L_{j}} r\right)}{k_{L_{j}} r}\right\} \sin \theta \\
& \left\{\bar{q}_{0}\right\}_{L_{j}}=-\frac{\pi}{k_{L_{j}}} \frac{N_{L_{j}}^{(1)} N_{L_{j}}^{(2)}}{\left(\frac{\partial F_{L}}{\partial k}\right)_{\omega, j}} \frac{H_{1}^{(2)}\left(k_{L_{j}} r\right)}{k_{L_{j}} r} \cos \theta \\
& \left\{\bar{w}_{0}\right\}_{L_{j}}=0
\end{aligned}
$$

where from equations (128) we have 


$$
N_{L_{j}}^{(2)}=1
$$

and

$$
N_{L_{j}}^{(1)}=i k_{L_{j}} \frac{\bar{L}}{2 \pi} \frac{1}{\left(A_{L}\right)_{11}}\left(A_{L_{s 1}}\right)_{11}
$$

In terms of the homogeneous notation we have

$$
\left[\frac{\dot{v}_{s}(D)}{\dot{v}_{0}}\right]_{H_{j}}=\left(A_{L_{s 1}}\right)_{11}
$$

and

$$
\left[\frac{\dot{v}_{n-1}}{\dot{v}_{0}}\right]_{H_{j}} \equiv\left[\frac{\dot{v}_{n}\left(\dot{z}_{n}-1\right)}{\dot{v}_{0}}\right]_{H_{j}}=\left(A_{L}\right)_{11}
$$

Thus, for the Love contribution

$$
\begin{aligned}
& \left\{\bar{v}_{0}\right\}_{L_{j}}=i \frac{\bar{L}}{2}\left[\frac{\dot{v}_{s}(D)}{\dot{v}_{0}}\right]_{H_{j}} \mathbf{A}_{L_{j}}\left\{H_{0}{ }^{(2)}\left(k_{L_{j}} r\right)-\frac{H_{1}{ }^{(2)}\left(k_{L_{j}} r\right)}{k_{L_{j}} r}\right\} \sin \theta \\
& \left\{\bar{q}_{0}\right\}_{L_{j}}=-i \frac{\bar{L}}{2}\left[\frac{\dot{v}_{s}(D)}{\dot{v}_{0}}\right]_{H_{j}} \mathbf{A}_{L_{j}} \frac{H_{1}{ }^{(2)}\left(k_{L_{j}} r\right)}{k_{L_{j}} r} \cos \theta
\end{aligned}
$$

where

$$
\mathbf{A}_{L_{j}}=\frac{1}{\left[\frac{\dot{v}_{n-1}}{\dot{v}_{0}}\right]_{H_{j}}\left(\frac{\partial F_{L}}{\partial k}\right)_{\omega, j}}
$$

As in previous sections the residue contributions are continuous through the source plane, and it follows that the residue displacements at depth for the horizontal point force are

$$
\begin{aligned}
&\left\{\bar{q}_{m}(r, \theta, z)\right\}_{R_{j}}=i\left[\frac{\dot{u}_{m}(z)}{\dot{w}_{0}}\right]_{H_{j}}\left\{\bar{w}_{0}\right\}_{R_{j}} \\
& \cdot\left\{H_{0}{ }^{(2)}\left(k_{R_{j}} r\right)-\frac{H_{1}{ }^{(2)}\left(k_{R_{j}} r\right)}{k_{R_{j}} r}\right\} / H_{1}{ }^{(2)}\left(k_{R_{j}} r\right) \\
&\left\{\bar{w}_{m}(r, \theta, z)\right\}_{R_{j}}=\left[\frac{\dot{w}_{m}(z)}{\dot{w}_{0}}\right]_{H_{j}}\left\{\bar{w}_{0}\right\}_{R_{j}}
\end{aligned}
$$

and

$$
\left\{\dot{v}_{m}(r, \theta, z)\right\}_{L_{j}}=\left[\frac{\dot{v}_{m}(z)}{\dot{v}_{0}}\right]_{H_{j}}\left\{\dot{v}_{0}\right\}_{L_{j}}
$$


for all $\mathrm{m}$. The homogeneous ratios are given for Rayleigh in terms of $A_{R_{m}}(z)$ as before. The Love ratios are similarly given in terms of

$$
A_{L_{m}}(z)=\mathfrak{Q}_{L_{m}}(z) \mathfrak{Q}_{L_{m-1}} \cdots \mathfrak{Q}_{L_{1}}
$$

where $Q_{L_{m}}(z)$ is the matrix for a sublayer in $m$ of thickness $d_{m}=z-z_{m-1}$.

In the remainder of this paper, we will be interested in Rayleigh and Love waves at large $r$. Neglecting terms in $r^{-3 / 2}$ which are small compared to terms in $r^{-1 / 2}$, we have as the dominant surface displacements,

$$
\begin{aligned}
\left\{\bar{w}_{0}\right\}_{R_{j}} & =i \frac{\bar{L}}{2}\left[\frac{\dot{u}_{s}^{*}(D)}{\dot{w}_{0}}\right]_{H_{j}} \mathbf{A}_{R_{j}} H_{1}^{\left({ }^{(2)}\right.}\left(k_{R_{j}} r\right) \cos \theta \\
\left\{\bar{q}_{0}\right\}_{R_{j}} & =-i \frac{\bar{L}}{2}\left[\frac{\dot{u}_{0}{ }^{*}}{\dot{w}_{0}}\right]_{H_{j}}\left[\frac{\dot{u}_{s}^{*}(D)}{\dot{w}_{0}}\right]_{H_{j}} \mathbf{A}_{R_{j}} H_{0}{ }^{(2)}\left(k_{R_{j}} r\right) \cos \theta \\
\left\{\bar{v}_{0}\right\}_{L_{j}} & =i \frac{\bar{L}}{2}\left[\frac{\dot{v}_{s}(D)}{\dot{v}_{0}}\right]_{H_{j}} \mathbf{A}_{L_{j}} H_{0}^{(2)}\left(k_{L_{j}} r\right) \sin \theta
\end{aligned}
$$

Up to here we have only considered sources with the restricted cylindrical geoometry assumed in equations ( 7 ). Because of the linear independence of $\sin l \theta$ and $\cos l \theta$ and the linear independence of angular solutions of different $l$, the generalizato point sources of more complicated $\theta$-dependence is straightforward. This assumes of course that the $r$ and $\theta$ dependence of the point source can be expressed as an integral or sum of integrals with integrands consisting of separable cylindrical solutions. In fact, using the results of the previous sections, the solutions for more complicated sources of this type can be found by inspection with the exception of source integrands involving $\delta \tau_{L_{s}}$, which were not included in this paper.

\section{Extension of the Horizontal Point Force Solutions to a Model of a Vertical Strike Fadlt}

In this section, we take the solutions for a horizontal point force at depth in a multilayered medium and extend them by spatial integration to represent a model for a vertical strike fault. This method of integration over a finite vertical fault plane was used by Ben-Menahem (1961). For Rayleigh waves he used the horizontal point force solutions of an elastic half-space and for the Love waves the corresponding solutions for one layer over a half-space. In this multilayered formulation, the vertical integration is evaluated exactly. For the time lagged integration over the horizontal fault dimension, we use the approximate evaluation given by BenMenahem.

Now consider in-phase horizontal point forces distributed continuously on a vertical $z$ axis for the interval $h_{1} \leqq z \leqq h_{2}$. The strength is adjusted so that as $\Delta h=h_{2}-h_{1}$ approaches zero, the integrated effect reverts to the point force solution at $z=h_{1}=h_{2}$. Then using equations (139), we obtain for a vertical segment of horizontal forces in a single source layer $s$, 


$$
\left\{\bar{w}_{0}\right\}_{R_{i}}^{\Delta h}=i \frac{\bar{L}}{2} \mathbf{A}_{R_{j}} H_{1}{ }^{(2)}\left(k_{R_{j}} r\right) \cos \theta \frac{1}{\Delta h} \int_{h_{1}}^{h_{2}}\left[\frac{\dot{u}_{s}{ }^{*}(h)}{\dot{w}_{0}}\right]_{H_{j}} d h
$$

where

$$
\left[\frac{\dot{u}_{s}^{*}(h)}{\dot{w}_{0}}\right]_{H_{j}}=\left[A_{R_{s}}(h)\right]_{12}^{*}+\left[\frac{\dot{u}_{0}^{*}}{\dot{w}_{0}}\right]_{H_{j}}\left[A_{R_{s}}(h)\right]_{11}
$$

and $\left[u_{0}^{*} / w_{0}\right]_{\mu_{j}}$ is a function of the layer array and not $h$.

Now since a given layer can be divided into as many sublayers as desired by the matrix relations (15), we can write

$$
A_{R_{s}}(h)=Q_{R_{8}}(l) A_{R_{s}}\left(h_{1}\right) \quad \text { where } l=h-h_{1}
$$

Performing the integration, we obtain

$$
\begin{aligned}
\int_{h_{1}}^{h_{2}}\left[\frac{\dot{u}_{s}^{*}(h)}{\dot{w}_{0}}\right]_{H_{j}} d h=\left[\frac{\dot{u}_{s}^{*}\left(h_{1}\right)}{\dot{w}_{0}}\right]_{H_{j}} I_{11}(\Delta h)+\left[\frac{\dot{w}_{s}\left(h_{1}\right)}{\dot{w}_{0}}\right]_{H_{j}} I_{12}^{*}(\Delta h) \\
+\left[\frac{\sigma_{s}^{*}\left(h_{1}\right)}{\frac{\dot{w}_{0}}{c}}\right]_{H_{j}} I_{13}(\Delta h)+\left[\frac{\tau_{R_{s}}\left(h_{1}\right)}{\frac{\dot{w}_{0}}{c}}\right]_{H_{j}} I_{14}^{*}(\Delta h)
\end{aligned}
$$

where

$$
\begin{aligned}
& I_{11}(\Delta h)=\int_{0}^{\Delta h}\left[\mathscr{Q}_{R_{s}}(l)\right]_{11} d l=\gamma_{s} \frac{\sin \Delta P_{s}}{k r_{\alpha_{s}}}-\left(\gamma_{s}-1\right) \frac{\sin \Delta Q_{s}}{k r_{\beta_{s}}} \\
& I_{12}^{*}(\Delta h)=\int_{0}^{\Delta h}\left[\mathscr{Q}_{R_{s}}(l)\right]_{12}^{*} d l=\left(\gamma_{s}-1\right) \frac{1}{k_{R_{j}} r_{\alpha_{s}}^{2}}\left(1-\cos \Delta P_{s}\right) \\
& +\frac{\gamma_{s}}{k_{R_{j}}}\left(1-\cos \Delta Q_{s}\right) \\
& I_{13}(\Delta h)=\int_{0}^{\Delta h}\left[Q_{R_{s}}(l)\right]_{13} d l=-\frac{1}{\rho_{s} c_{R_{j}}^{2}}\left[\frac{\sin \Delta P_{s}}{k_{R_{j}} r_{\alpha_{s}}}-\frac{\sin \Delta Q_{s}}{k_{R_{j}} r_{\beta_{s}}}\right] \\
& I_{14}^{*}(\Delta h)=\int_{0}^{\Delta h}\left[\mathscr{Q}_{R_{s}}(l)\right]_{14}^{*} d l=\frac{1}{\rho_{s} c_{R_{j}}^{2}}\left[\frac{1}{k_{R_{j}} r_{\alpha_{s}}^{2}}\left(1-\cos \Delta P_{s}\right)\right. \\
& \left.+\frac{1}{k_{R_{j}}}\left(1-\cos \Delta Q_{s}\right)\right]
\end{aligned}
$$

$\Delta P_{s}=k_{R_{j}} r_{\alpha_{s}} \Delta h$ and $\Delta P_{s}=k_{R_{j}} r_{\beta_{s}} \Delta h$

When the line of sources extends through the interface between layers say $s_{k}: k$ $=1, m$, we generalize our result to

$$
\left\{\bar{w}_{0}\right\}_{R_{j}}^{\Delta h}=i \frac{\bar{L}}{2} \mathbf{A}_{R_{j}} O_{R_{s}} H_{1}^{(2)}\left(k_{R_{j}} r\right) \cos \theta
$$


where

$$
\begin{aligned}
O_{R_{s}}=\frac{1}{\Delta h}\left\{\int_{h_{1}}^{z_{s_{1}}}\left[\frac{\dot{u}_{s_{1}}^{*}(h)}{\dot{w}_{0}}\right]_{H_{j}} d h+\sum_{k_{k=2}}^{m-1} \int_{z_{s_{k-1}}}^{z_{s_{k}}}\left[\frac{\dot{u}_{s_{k}}^{*}(h)}{\dot{w}_{0}}\right]_{H_{j}} d h\right. & \\
& \left.+\int_{z_{s_{m-1}}}^{h_{2}}\left[\frac{\dot{u}_{s_{m}}^{*}(h)}{\dot{w}_{0}}\right]_{H_{j}} d h\right\}
\end{aligned}
$$

The $1 / \Delta h$ is carried outside the summation, instead of $1 / \Delta h_{s_{k}}$ with each element, so that if all the layers were the same material the result would be consistent with a single layer. Similarly

$$
\left\{\bar{q}_{0}\right\}_{R_{j}}^{\Delta h}=-i \frac{\bar{L}}{2}\left[\frac{{\dot{u_{0}}}^{*}}{\dot{w}_{0}}\right]_{H_{j}} \mathbf{A}_{R_{j}} O_{R_{s}} H_{0}^{(2)}\left(k_{R_{j}} r\right) \cos \theta
$$

Now for Love waves, the integrated effect for a single source layer is

$$
\left\{\bar{v}_{0}\right\}_{L_{j}}^{\Delta h_{j}}=i \frac{\bar{L}}{2} \mathbf{A}_{L_{j}} H_{0}^{\left({ }^{2}\right)}\left(k_{L_{j}} r\right) \sin \theta \frac{1}{\Delta h} \int_{h_{1}}^{h_{2}}\left[\frac{\dot{v}_{s}(h)}{\dot{v}_{0}}\right]_{H_{i}} d h
$$

where

$$
\left[\frac{\dot{v}_{s}(h)}{\dot{v}_{0}}\right]_{H_{j}}=\left[A_{L_{s}}(h)\right]_{11}
$$

Decomposing $A_{L_{s}}(h)$ to

$$
A_{L_{s}}(h)=\mathfrak{a}_{L_{s}}(l) A_{L_{s}}\left(h_{1}\right)
$$

where

$$
l=h-h_{1}
$$

the integration yields

$$
\int_{h_{1}}^{h_{2}}\left[\frac{\dot{v}_{s}(h)}{\dot{v}_{0}}\right]_{H_{j}} d h=\left[\frac{\dot{v}_{s}\left(h_{1}\right)}{\dot{v}_{0}}\right]_{H_{j}} I_{11}(\Delta h)-\left[\frac{\tau_{L_{s}}^{*}\left(h_{1}\right)}{\frac{\dot{v}_{0}}{c}}\right]_{H_{j}} I_{12}^{*}(\Delta h)
$$

where

$$
\begin{aligned}
& I_{11}(\Delta h)=\int_{0}^{\Delta h}\left[Q_{L_{s}}(l)\right]_{11} d l=\frac{\sin \Delta Q_{s}}{k_{L_{j}} r_{\beta_{s}}} \\
& \left.I_{12}^{*}(\Delta h)=\int_{0}^{\Delta h}\left[Q_{L_{s}}(l)\right)\right]_{12}^{*} d l=\frac{1}{\mu_{s} k_{L_{j}} r_{\beta_{s}}}\left(1-\cos \Delta Q_{s}\right)
\end{aligned}
$$

When the segment extends vertically through many layers, we generalize to 


$$
\begin{aligned}
O_{L_{s}}=\frac{1}{\Delta h}\left\{\int_{h_{1}}^{z_{s_{1}}}\left[\frac{\dot{v}_{s_{1}}(h)}{\dot{v}_{0}}\right]_{H_{j}} d h+\sum_{k=2}^{m-1} \int_{z_{s_{k-1}}}^{z_{s_{k}}}\left[\frac{\dot{v}_{s_{k}}(h)}{\dot{v}_{0}}\right]_{H_{j}} d h\right. \\
\left.+\int_{z_{s_{m-1}}}^{h_{2}}\left[\frac{\dot{v}_{s_{m}}(h)}{\dot{v}_{0}}\right]_{H_{j}} d h\right\}
\end{aligned}
$$

and obtain

$$
\left\{\bar{v}_{0}\right\}_{L_{j}}^{\Delta h}=i \frac{L}{2} \mathbf{A}_{L_{j}} H_{0}{ }^{(2)}\left(k_{L_{j}} r\right) O_{L_{s}} \sin \theta
$$

We now shift the vertical line of horizontal forces in the horizontal direction from 0 to $b$ with velocity $v$. We take into consideration the necessary phase shift corresponding to a time shift in the source time function at each source point. We obtain

$$
\begin{aligned}
\left\{\bar{q}_{0}\right\}_{R_{j}}^{\Delta h, b} & =-i \frac{\bar{L}}{2}\left[\frac{\dot{u}_{0}^{*}}{\dot{v}_{0}}\right]_{H_{j}} \mathbf{A}_{R_{j}} O_{R_{s}} J_{0 R}^{c} \\
\left\{\tilde{w}_{0}\right\}_{R_{j}} & =i \frac{\bar{L}}{2} \mathbf{A}_{R_{j}} O_{R_{s}} J_{1 R}^{c}
\end{aligned}
$$

where by Ben-Menahem (1961)

$$
\begin{aligned}
J_{0 R}^{c} & =\frac{1}{b} \int_{0}^{b} \cos \theta H_{0}^{(2)}\left(k_{R_{j}} r\right) e^{-i \xi / v} d \xi \\
& \cong \frac{\cos \theta_{0}}{\left(k_{R_{j}} r\right)^{\frac{1}{2}}}\left(\frac{2}{\pi}\right)^{\frac{1}{2}}\left\{\frac{\sin X_{R}}{X_{R}}\right\} e^{-i\left(\omega \frac{r_{0}}{c_{R_{j}}}+X_{R}-\frac{\pi}{4}\right)}
\end{aligned}
$$

and

$$
\begin{aligned}
J_{1 R}^{c} & =\frac{1}{b} \int_{0}^{b} \cos \theta H_{1}{ }^{(2)}\left(k_{R_{j}} r\right) e^{-i \xi / v} d \xi \\
& \cong \frac{\cos \theta_{0}}{\left(k_{R_{j}} r\right)^{\frac{1}{2}}}\left(\frac{2}{\pi}\right)^{\frac{1}{2}}\left\{\frac{\sin X_{R}}{X_{R}}\right\} e^{-i\left(\frac{\omega r_{0}}{c_{R_{j}}}+x_{R}-\frac{3 \pi}{4}\right)}
\end{aligned}
$$

where

$$
X_{R}=\frac{\omega b}{c_{r_{j}}}\left(\frac{c_{R_{j}}}{v}-\cos \theta_{0}\right) \text { and } \quad c_{R_{i}}=\frac{\omega}{k_{R_{j}}}
$$

For the Love wave contribution, we obtain

$$
\left\{\bar{v}_{0}\right\}_{L_{j}}^{\Delta h, b}=i \frac{\bar{L}}{2} \mathbf{A}_{L_{j}} O_{L_{s}} J_{0 L}^{s}
$$


where

$$
\begin{aligned}
J_{0 L}^{8} & =\frac{1}{b} \int_{0}^{b} \sin \theta H_{0}^{(2)}\left(k_{L_{j}} r\right) e^{-i \xi / v} d \xi \\
& \cong \frac{\sin \theta_{0}}{\left(k_{L_{j}} r\right)^{\frac{1}{2}}}\left(\frac{2}{\pi}\right)^{\frac{1}{2}}\left\{\frac{\sin X_{L}}{X_{L}}\right\} e^{-i\left(\frac{\omega r_{0}}{c_{L_{j}}}+x_{L}-\frac{\pi}{4}\right)}
\end{aligned}
$$

and

$$
X_{L}=\frac{\omega b}{c_{L_{j}}}\left(\frac{c_{L_{j}}}{v}-\cos \theta_{0}\right) \quad \text { and } \quad c_{L_{j}}=\frac{\omega}{k_{L_{j}}}
$$

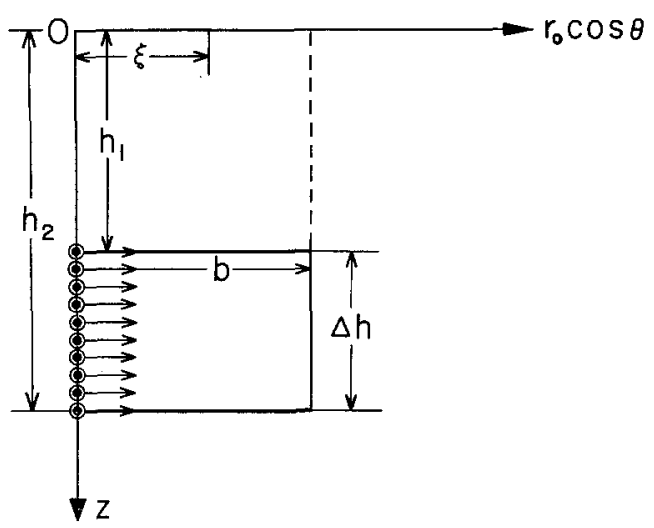

Frg. 3. Realization of vertical fault-plane.

The source fault plane geometry, used in the above evaluation, is the same as in Ben-Menahem except that ours is a right-hand coordinate system with $\theta$ being the negative of his (figures 3 and 4). The $r$ and $\theta$ in the integrals are given by

$$
\begin{aligned}
& r=r_{0}\left[1-2\left(\frac{\xi}{r_{0}}\right) \cos \theta_{0}+\left(\frac{\xi}{r_{0}}\right)^{2}\right]^{\frac{1}{2}} \\
& \theta=\cos ^{-1}\left\{\frac{r_{0} \cos \theta_{0}-\xi}{r}\right\}
\end{aligned}
$$

The evaluation of the integrals is approximate and based on the assumption that the range $r$ is large compared to the wavelength of the surface waves and the horizontal extent of the fault or

$$
\begin{aligned}
& r_{0} \gg k_{R_{j}}^{-1}, k_{L_{j}}^{-1} \\
& r_{0} \gg b
\end{aligned}
$$




$$
\begin{aligned}
& \left\{\bar{q}_{0}\right\}_{R_{j}}^{\Delta h, b}=-i\left(\frac{2}{\pi r_{0}}\right)^{\frac{1}{2}} \cos \theta_{0} \frac{\bar{L}}{2} k_{R_{j}}^{-\frac{1}{2}}\left[\frac{\dot{u}_{0}}{\dot{w}_{0}}\right]{ }_{H_{j}} O_{R_{s}} \mathbf{A}_{R_{j}}\left\{\frac{\sin X_{R}}{X_{R}}\right\} e^{-i\left(\frac{\omega r_{0}}{c_{R_{j}}}+x_{R}-\frac{\pi}{4}\right)} \\
& \left\{\bar{w}_{0}\right\}_{R_{j}}^{\Delta h, b}=i\left(\frac{2}{\pi r_{0}}\right)^{\frac{1}{2}} \cos \theta_{0} \frac{\bar{L}}{2} k_{R_{j}}^{-\frac{1}{2}} O_{R_{s}} \mathbf{A}_{R_{j}}\left\{\frac{\sin X_{R}}{X_{R}}\right\} e^{-i\left(\frac{\omega r_{0}}{c_{R_{j}}}+X_{R}-\frac{3 \pi}{4}\right)} \\
& \left\{\bar{v}_{0}\right\}_{L_{j}}^{\Delta h, b}=i\left(\frac{2}{\pi r_{0}}\right)^{\frac{1}{2}} \sin \theta_{0} \frac{L}{2} k_{R_{j}}^{-\frac{1}{2}} O_{L_{s}} \mathbf{A}_{L_{i}}\left\{\frac{\sin X_{L}}{X_{L}}\right\} e^{-i\left(\frac{\omega r_{0}}{c_{L_{j}}}+x_{L}-\frac{\pi}{4}\right)}
\end{aligned}
$$

In addition we may extend our model to a strike slip fault by considering a point couple instead of a point force moving along the finite fault plane. We accomplish this by differentiating the point force results with respect to the horizontal coordinate $\eta$ perpendicular to the fault plane where

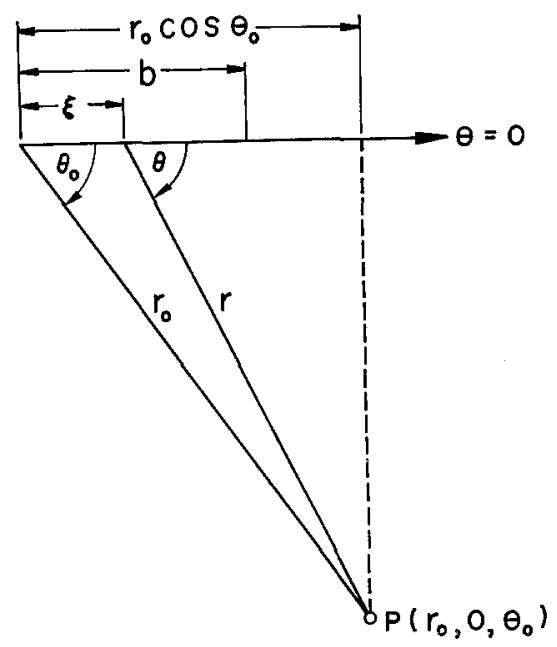

FIG. 4. Geometry of free surface.

$$
\frac{\partial}{\partial \eta}=\sin \theta_{0}\left(\frac{\partial}{\partial r_{0}}\right)+\frac{\cos \theta_{0}}{r_{0}}\left(\frac{\partial}{\partial \theta_{0}}\right)
$$

Fquation (156) applies strictly to the single horizontal couple or the vertical line of couples. However, to our order of approximation in the horizontal integration, it can also be applied to the horizontal moving couples on a finite fault. As a result, neglecting all but the lowest order of $r_{0}$, we obtain from equations (155)

$$
\begin{aligned}
& \left\{\bar{q}_{0}\right\}_{R_{j}}^{c, \Delta h, b}=-\left(\frac{2}{\pi r_{0}}\right)^{1 / 2} \sin \theta_{0} \cos \theta_{0} \frac{\bar{L}^{\prime}}{2} k_{R_{j}}^{1 / 2}\left[\frac{{\dot{u_{0}}}^{*}}{\dot{w}_{0}}\right]_{H_{j}} O_{R_{s}} \mathbf{A}_{R_{j}} \\
& \times\left\{\frac{\sin X_{R}}{X_{R}}\right\} e^{-i\left[\left(\omega r_{0} / c_{R_{j}}\right)+X_{R}-(\pi / 4)\right]} \\
& \left\{\bar{w}_{0}\right\}_{R_{j}}^{c, \Delta h, b}=\left(\frac{2}{\pi r_{0}}\right)^{1 / 2} \sin \theta_{0} \cos \theta_{0} \frac{\bar{L}^{\prime}}{2} k_{R_{j}}^{1 / 2} O_{R_{s}} \mathbf{A}_{R_{j}} \\
& \times\left\{\frac{\sin X_{R}}{X_{R}}\right\} e^{-i\left\lfloor\left(\omega r_{0} / c_{R j}\right)+X_{\left.R^{-}-(3 \pi / 4)\right]}\right.} \\
& \{\bar{v}\}_{L_{j}}^{c, \Delta h, b}=\left(\frac{2}{\pi r_{0}}\right)^{1 / 2} \sin ^{2} \theta_{0} \frac{\bar{L}^{\prime}}{2} k_{L_{j}}^{1 / 2} O_{L_{s}} \mathbf{A}_{L_{j}}\left\{\frac{\sin X_{L}}{X_{L}}\right\} e^{-i\left[\left(\omega r_{0} / c_{L_{j}}\right)+X_{L}-\pi / 4\right]}
\end{aligned}
$$


where $\bar{L}^{\prime}$, the time transformed couple strength has the dimensions of $\bar{L} \times$ length or force $X$ time $X$ length.

\section{Extension to a Surface Liquid Layer}

Now consider the same solid array with the addition of a liquid layer at the formerly free surface. To be consistent with the previous notation we denote the liquid layer as layer 0 . At the solid-liquid interface, $z_{0}$, we impose continuity of normal stress and vertical displacement. In addition we require that the tangential stress vanish at $z_{0}$, and that all stresses vanish at the free surface $z_{-1}$.

Solving for the surface wave contributions of the new array, we find that the solutions can be expressed in the same form as the previous all solid solutions given by equations $(85),(103),(134),(138)$, and (139). In order to use these solutions for the liquid-surface layer problem, we make the following substitutions

$$
\begin{aligned}
& F_{R}=[N K-L M]+[T][G N-L H] \\
& {[T]=\left[\frac{\sigma_{0}}{\frac{\dot{u}_{0}}{c}}\right]_{H}=i\left[T^{*}\right]=i \frac{\rho_{0} c^{2}}{r_{a_{0}}} \tan P_{0}}
\end{aligned}
$$

and replace the homogeneous ratios defined in terms of the Thomson-Haskell matrix elements by equations (80) and 82) with

$$
\begin{aligned}
& {\left[\frac{\dot{u}_{m}^{*}(z)}{\dot{u}_{0}}\right]_{H_{j}}=\left[A_{R_{m}}(z)\right]_{12}+\left[\frac{\dot{u}_{0}^{*}}{\dot{u}_{0}}\right]_{H_{j}}\left[A_{R_{m}}(z)\right]_{11}+\left[T^{*}\right]\left[A_{R_{m}}(z)\right]_{13}} \\
& {\left[\frac{\dot{w}_{m}(z)}{\dot{u}_{0}}\right]_{H_{j}}=\left[A _ { R _ { m } } ( z ) \left[22-\left[\frac{\dot{u}_{0}^{*}}{\dot{u}_{0}}\right]_{H_{j}}\left[A_{R_{m}}(z)\right]_{12}^{*}-\left[T^{*}\right]\left[A_{R_{m}}(z)\right]_{23}^{*}\right.\right.} \\
& {\left[\frac{\sigma_{R_{m}}^{*}(z)}{\frac{\dot{u}_{0}}{c}}\right]_{H_{j}}=\left[A_{R_{m}}(z)\right]_{32}+\left[\frac{{\dot{u_{0}}}^{*}}{\dot{w}_{0}}\right]_{H_{j}}\left[A_{R_{m}}(z)\right]_{31}+\left[T^{*}\right]\left[A_{R_{m}}(z)\right]_{33}} \\
& {\left[\frac{\frac{\tau_{R_{m}}(z)}{\dot{u}_{0}}}{c}\right]_{H_{j}}=\left[A_{R_{m}}(z)\right]_{42}-\left[\frac{{\dot{u_{0}}}^{*}}{\dot{u}_{0}}\right]_{H_{j}}\left[A_{R_{m}}(z)\right]_{41}^{*}-\left[T^{*}\right]\left[A_{R_{m}}(z)\right]_{43}^{*}} \\
& {\left[\frac{\dot{u}_{0}}{\left.\dot{u}_{0}\right]_{H_{j}}}=\frac{K}{L^{*}}-\frac{G^{*}}{L^{*}}\left[T^{*}\right]=-\frac{\left[K H-G^{*} M^{*}\right]}{\left[G^{*} N-L^{*} H\right]}\right.} \\
& {\left[\frac{\frac{\varphi_{m}(z)}{\dot{u_{0}}}}{c}\right]_{H_{j}}=\frac{\gamma_{m}}{k_{r j}^{2}}\left\{\left[\frac{\dot{u}_{m}^{*}(z)}{\dot{w}_{0}}\right]_{H_{j}}-\frac{1}{2 \mu_{m}}\left[\frac{\frac{\sigma_{m}{ }^{*}(z)}{\dot{u}_{0}}}{c}\right]_{H_{j}}\right\}}
\end{aligned}
$$

If we let $\left[T^{*}\right]=0$, expressions (158) and (159) return to our earlier expressions for an all solid array.

The above relations pertain to displacements and stresses in the solids normalized 
to the vertical displacement at the liquid-solid interface. Displacements and pressures in the liquid layer are given by

$$
\begin{aligned}
& {\left[\frac{\dot{u}_{0}^{*}(z)}{\dot{w}_{0}}\right]_{H_{j}}=-\frac{\sin \left\{k r_{a_{0}} d_{0}(z)\right\}}{r_{a_{0}} \cos P_{0}}} \\
& {\left[\frac{\dot{w}_{0}(z)}{\dot{w}_{0}}\right]_{H_{j}}=\frac{\cos \left\{k r_{a_{0}} d_{0}(z)\right\}}{\cos P_{0}}} \\
& {\left[\frac{\sigma_{0}^{*}(z)}{\dot{w}_{0}}\right]_{H_{j}}=-\rho_{0} c^{2}\left[\frac{\dot{u}_{0}(z)}{\dot{w}_{0}}\right]_{H_{j}}}
\end{aligned}
$$

where $d_{0}(z)=z-z_{-1}$ and $d_{0}=z_{0}-z_{-1}$.

The addition of the liquid layer has no effect on the Love surface wave contributions and their pertinent homogeneous ratios remain

$$
\begin{gathered}
{\left[\frac{\dot{v}_{m}(z)}{\dot{v}_{0}}\right]_{H_{j}}=\left[A_{L_{m}}(z)\right]_{11}} \\
{\left[\frac{\frac{\tau_{L_{m}}^{*}(z)}{\dot{v}_{0}}}{c}\right]_{H_{j}}=\left[A_{L_{m}}(z)\right]_{21}^{*}}
\end{gathered}
$$

Using equations (159), the adaption of the finite fault solutions to a liquid surface layer is straightforward and will not be given here.

\section{Discussion}

Almost all previous work on sources in layered elastic media has been restricted to simple layer models. The frequency domain displacements derived in this paper exhibit many of the characteristics inferred from the work on simple models. Obviously, the radial and azimuthal dependence are the same as obtained for simple models. Therefore the factors obtained for a model of a vertical strike slip fault which depend on $r$ and $\theta$ are identical to the ones obtained by Ben-Menahem (1960) for the same model. Their application to determining fault parameters such as fault length and rupture velocity is thoroughly described by Ben-Menahem and thus will not be discussed here.

We will restrict our discussion to the effects of source depth and layering on the spectral amplitude of displacements as derived in this paper. Jardetzky (1953) and Kellis-Borok (1953) have also given expressions for these effects in terms of ratios of determinants of order $(4 n-2)$. The significant difference in their formulation and the one given here, is that we are able to simplify the displacement expression so that the effects of layering, source depth and receiver depth are separated into independent factors.

For the various sources investigated, the factors are as follows:

1. Explosive source 


$$
\begin{gathered}
\{\bar{w}(z: D)\}_{R_{j}} \sim\left[\frac{\dot{w}_{r}(z)}{\dot{w}_{0}}\right]_{H_{j}}\left[\frac{\frac{\varphi_{s}(D)}{\dot{w}_{0}}}{c}\right]_{H_{j}} \mathbf{A}_{R_{j}} k_{R_{j}}^{1 / 2} \\
\{\bar{q}(z ; D)\}_{R_{j}} \sim\left[\frac{\dot{u}_{r}^{*}(z)}{\dot{w}_{0}}\right]_{H_{j}}\left[\frac{\frac{\varphi_{s}(D)}{\dot{w}_{0}}}{c}\right]_{H_{j}} \mathbf{A}_{R_{j}} k_{R_{j}}^{1 / 2}
\end{gathered}
$$

2. Vertical point force

$$
\begin{aligned}
& \{\bar{w}(z ; D)\}_{R_{j}} \sim\left[\frac{\dot{w}_{r}(z)}{\dot{w}_{0}}\right]_{H_{j}}\left[\frac{\dot{w}_{s}(D)}{\dot{w}_{0}}\right]_{H_{j}} \mathbf{A}_{R_{j}} k_{R_{j}}^{-1 / 2} \\
& \{\bar{q}(z ; D)\}_{R_{j}} \sim\left[\frac{\dot{u}_{r}^{*}(z)}{\dot{w}_{0}}\right]_{H_{j}}\left[\frac{\dot{w}_{s}(D)}{\dot{w}_{0}}\right]_{H_{j}} \mathbf{A}_{R_{j}} k_{R_{j}}^{-1 / 2}
\end{aligned}
$$

3. Horizontal point force

$$
\begin{aligned}
& \{\bar{w}(z ; D)\}_{R_{j}} \sim\left[\frac{\dot{w}_{r}(z)}{\dot{w}_{0}}\right]_{H_{j}}\left[\frac{\dot{u}_{s}^{*}(D)}{\dot{w}_{0}}\right]_{H_{j}} \mathbf{A}_{R_{j}} k_{R_{j}}^{-1 / 2} \\
& \{\bar{q}(z ; D)\}_{R_{j}} \sim\left[\frac{\dot{u}_{r}^{*}(z)}{\dot{w}_{0}}\right]_{H_{j}}\left[\frac{\dot{u}_{s}^{*}(D)}{\dot{w}_{0}}\right]_{H_{j}} \mathbf{A}_{R_{j}} k_{R_{j}}^{-1 / 2} \\
& \{\bar{v}(z ; D)\}_{L_{j}} \sim\left[\frac{\dot{v}_{r}(z)}{\dot{v}_{0}}\right]_{H_{j}}\left[\frac{\dot{v}_{s}(D)}{\dot{v}_{0}}\right]_{H_{j}} \mathbf{A}_{L_{j}} k_{L_{j}}^{-1 / 2}
\end{aligned}
$$

where the $r$ and $s$ subscripts in the $H_{j}$ subscripted brackets refer to receiver and source layers respectively. The $H_{j}$ subscripted quantities in equations (163) and (164) refer to the ratios of velocity or displacement with depth for the homogeneous case in the $j$ th mode. The homogeneous ratio of dilation, $\varphi_{s}$, with depth used in equations (162) can also be given in terms of the homogeneous ratios of horizontal displacement and normal stress with depth. In the vertical fault models, the source depth effect of equations (164) is replaced by the average value of the point force factor over the vertical dimension of the finite fault.

From equations (162), (163) and (164) we see that the relations between displacement and receiver depth, $z$, are the same regardless of the type of source considered here. This fact was pointed out by Kellis-Borok and Yanovskaya (1962) as being true for all sources. They failed to realize though that for vector point forces and the explosive source, the relations between displacement and source depth, $D$, for a given mode $j$ can be expressed by similar relations. From our results, we have as the source depth relations

1. Explosive source 


$$
\begin{aligned}
& \frac{\{\bar{w}(z ; D)\}_{R_{j}}}{\{\bar{w}(z ; 0)\}_{R_{j}}}=\frac{\{\bar{q}(z ; D)\}_{R_{j}}}{\{\bar{q}(z ; 0)\}_{R j}} \\
&=\left[\frac{\varphi_{s}(D)}{\varphi_{s}(0)}\right]_{H_{j}}\left(\frac{\beta_{s}}{\beta_{1}}\right)^{2}\left[\frac{\left(1-\frac{a_{1}^{2} k_{\beta_{1}}^{2}}{4}\right)^{2}+k_{\alpha_{1}}^{2} a_{1}^{2}}{\left(1-\frac{a_{s}^{2} k_{\beta_{s}}^{2}}{4}\right)+k_{\alpha_{s}}^{2} a_{s}^{2}}\right] \frac{\bar{p}_{0 s}}{\bar{p}_{01}}\left(\frac{a_{s}}{a_{1}}\right)^{3}
\end{aligned}
$$

2. Vertical point force

$$
\frac{\{\bar{w}(z ; D)\}_{R_{j}}}{\{\bar{w}(z ; 0)\}_{R_{j}}}=\frac{\{\bar{q}(z ; D)\}_{R_{j}}}{\{\bar{q}(z ; 0)\}_{R j}}=\frac{\{\bar{w}(D ; z)\}_{R_{j}}}{\{\bar{w}(0 ; z)\}_{R_{j}}}=\left[\frac{\dot{w}_{s}(D)}{\dot{w}_{0}}\right]_{H_{j}}
$$

3. Horizontal point force

$$
\begin{aligned}
& \left.\frac{\{\bar{w}(z ; D)\}_{R_{j}}}{\{\bar{w}(z ; 0)\}_{R_{j}}}=\frac{\{\bar{q}(z ; D)\}_{R_{j}}}{\{\bar{q}(z ; 0)\}_{R_{j}}}=\frac{\{\bar{q}(D ; z)\}_{R_{j}}}{\{\bar{q}(0 ; z)\}_{R_{j}}}=\left[\frac{\dot{u}_{s}^{*}(D)}{\dot{w}_{0}}\right]\right]_{H_{j}} /\left[\frac{\dot{u}_{0}^{*}}{\dot{w}_{0}}\right]_{H_{j}} \\
& \frac{\{\bar{v}(z ; D)\}_{L_{j}}}{\{\bar{v}(z ; 0)\}_{L_{j}}}=\frac{\{\bar{v}(D ; z)\}_{L_{j}}}{\{\bar{v}(0 ; z)\}_{L_{j}}}=\left[\frac{\dot{v}_{s}(D)}{\dot{v}_{0}}\right]_{H_{j}}
\end{aligned}
$$

For volume sources, not considered in this paper, Kellis-Borok and Yanovskaya (1962) give a set of formulas taken from M. G. Niegaus which allow one to calculate source depth relations. This is accomplished by performing integrations over the vertical coordinate of the homogeneous solutions weighted by the actual source forces.

Actually the source depth relations given in equations (166) and (167) could have been determined using theorems on scalar and elastic reciprocity given by, among others, Rayleigh (1877) and Knopoff and Gangi (1959). These theorems apply to the total motion but as seen from our results, they are also true for the surface wave contribution to the total motion. For Rayleigh waves we see that the vertical surface displacement at $A$ due to an internal horizontal point force at $B$ is equal to the horizontal displacement at $B$ due to a surface vertical point force of the same strength at $A$. Love waves obey scalar reciprocity in that the source and receiver depth factors are interchangeable.

Sherwood and Spencer (1962) using another reciprocity theorem by Rayleigh (1877) postulated that the surface displacement at $A$ due to an internal dilatational source at $B$ is identical to the dilatation at $B$ produced by a vertical point force at $A$. From our results we see that this is true if the magnitude of the force is properly normalized to the dilatation source, that is, if we set the coefficient of the radial part of the dilatation source in equation (41) equal to unity then our vertical force, $\bar{L}$, must be of magnitude $i 4 \pi \omega^{2} \rho_{s}$.

The factors $\mathbf{A}_{R}$ and $\mathbf{A}_{L}$ for Rayleigh and Love waves respectively are independent of source and receiver depth and also the type of source and receiver. They depend only on the properties of the layers in the array. From equations (103), $\mathbf{A}_{k} / 2$ can be considered as the spectral vertical response of Rayleigh waves at the free surface 
to a unit vertical surface point force after removing the transmission effect in the $r$ direction. Similarly from equations (137), $\mathbf{A}_{L} / 2$ is the spectral $S H$ response of Love waves at the free surface to a unit horizontal surface point force.

Computer programs based on the Thomson-Haskell matrices for the computation of dispersion $(c, T)$ in multilayered half-spaces yield the homogeneous motion stress ratios as a by product. Several such programs are described in the literature. Using a technique described in Part II to calculate analytic $(\partial F / \partial k)_{\omega}$, it is therefore possible to modify these programs to compute analytically values of the layering effect, $\mathbf{A}_{R}$ and $\mathbf{A}_{L}$, as a function of phase velocity or frequency.

The obvious advantages of the factoring is that the homogeneous stress and displacement ratios, and the layering effect need be calculated only once for a given frequency and mode. Once these quantities are plotted, they can easily be used to calculate the spectrum under many different conditions of source type, source depth, and receiver depth, without requiring the use of a large computer.

The source theory presented here is the ground work for the numerical computation of amplitude spectra and synthetic seismograms in later papers. There spectra and seismograms will be calculated under various conditions of source type, structure and source depth. Then by comparing spectra and seismograms, we will see if the various parameters have quantitative or qualitative character in the frequency or time domain.

Theoretical seismograms of continental Rayleigh and Love surface waves demonstrating the effect of distance and source depth for a horizontal couple have already been calculated as part of the research described here (Harkrider, 1963). Similar calculations are now in progress for an oceanic structure.

\section{ACKNOWLEDGMENTS}

This research was supported by Grant No. AF-AFosR-25-63 of the Air Force Office of Scientific Research as part of the Advanced Research Projects Agency Project veca. The author is grateful to Professor Frank Press for his support and encouragement throughout this study. Many valuable discussions were held with Drs. D. L. Anderson, R. A. Phinney and C. B. Archambeau. Their cooperation and support is acknowledged with gratitude.

\section{ReFERENCES}

Ben-Menahem, A.

1961. "Radiation of seismic surface-waves from finite moving sources," Bull. Seism. Soc. Am., 51, 401-435.

Blake, F. G., Jr.

1952. "Spherical wave propagation in solid media," Journ. Acoust. Soc. Amer., 24, 211-215.

Dorman, J., M. Ewing, and J. Oliver

1960. "Study of shear velocity distribution by mantle Rayleigh waves," Bull. Seism. Soc. Am., 50, 87-115.

Dorman, J., and D. Prentiss

1960. "Particle Amplitude Profiles for Rayleigh Waves on a Heterogeneous Earth," $J$. Geophys. Res., 65, 3805-3816.

Dorman, J.

1962. "Period equation for waves of Rayleigh type on a layered, liquid-solid half space," Bull. Seism. Soc. Am., 52, 389-397.

Ewing, J., W. Jardetzky, and F. Press

1957. Elastic Waves in Layered Media, McGraw-Hill Book Company, New York.

Fu, C. Y. 
1945. "On the origin and energy of oscillatory earthquake waves, Bull. Seism. Soc. Am., $35,37-42$.

Gilbert, F., Jr.

1956. "Seismic wave propagation in a two-Iayer half-space," Thesis, Massachusetts Institute of Technology.

Gilbert, F., Jr., and G. J. F. MacDonald

1960. "Free oscillations of the earth, 1, Toroidal oscillations," J. Geophys. Research, 65, $675-693$.

Harkrider, D. G., and D. L. Anderson

1962. "Computation of surface wave dispersion for multilayered anisotropic media," Bull. Seism. Soc. Am., 52, 321-332.

Harkrider, D. G.

1963. "Theoretical Love and Rayleigh waves from a vertical strike-slip fault model at depth in a multilayered half-space," Abstracts of Papers, III, XIII General Assembly of I. U. G. G. Berkeley, California.

Haskell, N. A.

1953. "The dispersion of surface waves on multilayered media," Bull. Seism. Soc, Am., 43, $17-34$.

1962. "Crustal Reflection of Plane P and SV Waves," J. Geophys. Research, 67, 4751-4767.

Jardetzky, W. S.

1953. "Period equation for an $n$-layered half-space and some related questions," Columbia Univ. Lamont Geol. Obs. Tech. Rept. Seismology, 29.

Kawasumi, H., and R. Yosiyama

1935. "On an elastic wave animated by the potential energy of initial strain," Bull. Earthquake Res. Inst., Tokyo, 13, 496-503.

Kellis-Borok, V. L.

1953. "Interference seismic waves in a layered median," Thesis, Inst. of Physics of the Earth, Acad. Sci. U.S.S.R.

Kellis-Borok, V. I., and T. B. Yanovskaya

1962. "Dependence of the spectrum of surface waves on the depth of the focus within the earth's crust," Isv. Akad. Nauk. SSSR, Geophys. Ser., 1532-1539.

Knopoff, L., and A. F. Gangi

1959. "Seismic Reciprocity," Geophysics, 24, 681-691.

Menzel, H.

1957. "Uber des spektrum seismischer Wellen, die durch Sprengungen erzeugt werden," Annali di Geofisica, 4, 301-321.

Pekeris, C. L

1955. "The seismic Buried Pulse," Proc. Nat. Acad. Sci., U.S., 41, 629-639.

Press, F., D. Harkrider, and C. A. Seafeldt

1961. "A fast, convenient program for computation of surface-wave dispersion curves on multilayered media," Bull. Seism. Soc. Am., 51, 495-502.

Rayleigh, Lord

1877. The Theory of Sound, 1. Dover Publications, New York.

Sharpe, J. A

1942. "The production of elastic waves by explosive pressures," Geophysics, 7, 144-154.

Sherwood, J. W. C., and T. W. Spencer

1962. "Signal-to-noise ratio and spectra of explosion-generated Rayleigh waves," Bull. Seism. Soc. Am., 52, 573-594.

Sneddon, J.

1950. Fourier Transforms, McGraw-Hill Book Company, New York.

Thomson, W. T.

1950. "Transmission of elastic waves through a stratified solid medium," Jour. Appl. Phys., 21, 89. 
Yanovskaya, T. B.

1958. "On the determination of the dynamic parameters of the focus hypocenter of an earthquake from records of surface waves, Isv. Akad. Nauk, SSSR, Geophys. Ser., 289-201.

Seismological Laboratory

California Institute of Teghnology

Pasadena, California

Division of the Geological Sciences

Contribution No. 1218

Manuscript received November 11, 1963. 